\title{
Pathogenomic analyses of Shigella isolates inform factors limiting shigellosis prevention and control across LMICs
}

\author{
Rebecca J. Bengtsson ${ }^{1}$, Adam J. Simpkin², Caisey V. Pulford ${ }^{1,3}$, Ross Low ${ }^{4}$, David A. Rasko ${ }^{5}{ }^{5}$, \\ Daniel J. Rigden², Neil Hall ${ }^{4,6}$, Eileen M. Barry ${ }^{7}{ }^{7}$, Sharon M. Tennant ${ }^{7}$ and Kate S. Baker ${ }^{1 凶}$
}

\begin{abstract}
Shigella spp. are the leading bacterial cause of severe childhood diarrhoea in low- and middle-income countries (LMICs), are increasingly antimicrobial resistant and have no widely available licenced vaccine. We performed genomic analyses of 1,246 systematically collected shigellae sampled from seven countries in sub-Saharan Africa and South Asia as part of the Global Enteric Multicenter Study (GEMS) between 2007 and 2011, to inform control and identify factors that could limit the effectiveness of current approaches. Through contemporaneous comparison among major subgroups, we found that $S$. sonnei contributes $\geq 6$-fold more disease than other Shigella species relative to its genomic diversity, and highlight existing diversity and adaptative capacity among $S$. flexneri that may generate vaccine escape variants in $<6$ months. Furthermore, we show convergent evolution of resistance against ciprofloxacin, the current WHO-recommended antimicrobial for the treatment of shigellosis, among Shigella isolates. This demonstrates the urgent need to integrate existing genomic diversity into vaccine and treatment plans for Shigella, providing a framework for the focused application of comparative genomics to guide vaccine development, and the optimization of control and prevention strategies for other pathogens relevant to public health policy considerations.
\end{abstract}

S higellosis is a diarrhoeal disease responsible for approximately 212,000 annual deaths and accounting for $13.2 \%$ of all diarrhoeal deaths globally ${ }^{1}$. The Global Enteric Multicenter Study (GEMS) was a large case-control study conducted between 2007 and 2011, investigating the aetiology and burden of moderate-to-severe diarrhoea (MSD) in children $<5$ years old in low- and middle-income countries (LMICs) ${ }^{2}$. GEMS revealed shigellosis as the leading bacterial cause of diarrhoeal illness in children, who represent a major target group for vaccination ${ }^{3}$. The aetiological agents are Shigella, a Gram-negative genus comprising S. flexneri, S. sonnei, S. boydii and S. dysenteriae, with the former two species causing the majority (90\%) of attributable shigellosis in children in LMICs ${ }^{3}$. Currently, the disease is primarily managed through supportive care and antimicrobial therapy. However, there has been an increase in antimicrobial resistance (AMR) among Shigella $a^{4}$. Particularly concerning is the rise in resistance against the fluoroquinolone antimicrobial ciprofloxacin, the current World Health Organization (WHO)-recommended treatment, such that fluoroquinolone-resistant (FQR) Shigella is one of a dozen pathogens for which WHO notes new antimicrobial therapies are urgently needed $^{5}$. The disease burden and increasing AMR of Shigella call for improvements in treatment and management options for shigellosis, and substantial momentum has built to rise to this challenge.

However, there is no licenced vaccine widely available for Shigella and one of the main challenges in its development is the considerable genomic and phenotypic diversity of the organisms ${ }^{6}$.
The distinct lipopolysaccharide O-antigen structures of Shigella determine its serotype and is responsible for conferring the shortto medium-term serotype-specific immunity following infec$\operatorname{tion}^{7-10}$. Hence, considerable efforts are focused on generating $\mathrm{O}$-antigen-specific vaccines. However, except for $S$. sonnei that has a single serotype, all species encompass multiple diverse serotypes: 14 serotypes/subserotypes for S. flexneri, 19 for $S$. boydii and 15 for $S$. $d y$ senteriae $^{11}$. Thus, for serotype-targeted vaccine approaches, multivalent vaccines are proposed to provide broad protection against the disease $^{12}$. While $\mathrm{O}$-antigen conjugates are a leading strategy, challenge studies have recently demonstrated poor clinical efficacy ${ }^{13,14}$. An attractive alternative and/or complement to serotype-targeted vaccine formulations are specific-subunit vaccines that target highly conserved proteins and may offer broad protection. There are several candidates in development that have demonstrated protection in animal models ${ }^{15,16}$, but the degree of antigenic variation in these targets among the global Shigella population remains unknown. Other strategies being explored include vaccines combining protein and serotype antigens, such as Generalized Modules of Membrane Antigens (GMMA), which involves use of outer membrane particles derived from genetically modified $S$. sonnei to elicit a stronger immune response ${ }^{17}$. However, GMMA also failed to demonstrate clinical efficacy against shigellosis in a recent challenge study ${ }^{18}$, indicating the continuing challenges of Shigella vaccinology.

Whole-genome sequencing analysis (WGSA) provides sufficient discriminatory power to resolve phylogenetic relationships and

\footnotetext{
${ }^{1}$ Clinical Infection, Microbiology and Immunity, Institute of Infection, Veterinary and Ecological Sciences, The University of Liverpool, Liverpool, UK. ${ }^{2}$ Biochemistry and Systems Biology, Institute of Systems, Molecular and Systems Biology, The University of Liverpool, Liverpool, UK. ${ }^{3}$ Gastrointestinal Infections and Food Safety (One Health), United Kingdom Health Security Agency, London, UK. ${ }^{4}$ Earlham Institute, Norwich Research Park, Norwich, UK. ${ }^{5}$ Department of Microbiology and Immunology, Institute for Genome Sciences, University of Maryland School of Medicine, Baltimore, MD, USA. ${ }^{6}$ School of Biological Sciences, University of East Anglia, Norwich, UK. ${ }^{7}$ Center for Vaccine Development and Global Health, University of Maryland School of Medicine, Baltimore, MD, USA. 䒽e-mail: kbaker@liverpool.ac.uk
} 
characterize the diversity of bacterial pathogens, which are essential to informing vaccine development and other aspects of disease control ${ }^{19,20}$. However, these critical analysis tools are yet to be applied to a pathogen collection appropriate for broadly informing shigellosis control in the critical demographic of children in LMICs. Here we apply WGSA to Shigella isolates sampled during GEMS, representing 1,246 systematically collected isolates from across seven nations in sub-Saharan Africa and South Asia with some of the highest childhood mortality rates ${ }^{2,21}$. We found evidence of the potential benefit of genomic subtype-based targeting, characterized pathogen features that will complicate current vaccine approaches, and highlighted regional differences in Shigella diversity, as well as determinants of AMR, including convergent evolution towards resistance against currently recommended treatments. Our analysis of this unparalleled pathogen collection informs the control and prevention of shigellosis in those populations most vulnerable to the disease.

\section{Regional diversity of Shigella spp. across LMICs}

To date, this is the largest representative dataset of Shigella genomes from LMICs $(n=1,246)$, collected across seven sites from Asia, West Africa and East Africa, comprising 806 S. flexneri, 305 S. sonnei, 75 S. boydii and 60 S. dysenteriae (Fig. 1a). To compare the genomic diversity of Shigella species, we determined the distributions of pairwise single-nucleotide polymorphism (SNP) distances and scaled the total detected SNPs against the length of the chromosome (in kbp) for each species (Fig. 1b). This revealed that $S$. boydii contained the greatest diversity (24.2 SNPs per kbp), followed by S. flexneri (19.5 SNPs per kbp) and S. dysenteriae (11.8 SNPs per $\mathrm{kbp}$ ), with $S$. sonnei being $>9.8$-fold less diverse (1.2 SNPs per kbp) or $>13.1$-fold less diverse (0.9 SNPs per $\mathrm{kbp}$ ) when excluding two outliers (see below, Fig. 1b). Thus, S. sonnei caused more disease relative to genomic diversity than $S$. flexneri (5.9-fold), S. dysenteriae (497.5-fold) or S. boydii (99.5-fold) (Fig. 1b). However, when stratified by serotype/subserotype or genomic subtype, S. sonnei had a more comparable diversity and less pronounced increase in disease burden relative to genomic diversity (1.1-22.1-fold higher by serotype/subserotype and 1.2-4.9-fold higher by genomic subtype) (Supplementary Figs. 1 and 2). Further analyses revealed that the reduced diversity of $S$. sonnei (measured in chromosomal SNPs) was also reflected by a reduced accessory genome repertoire (Extended Data Fig. 1) and less recombination events across the genomes (Extended Data Fig. 2) relative to other species. This indicates the value of vaccination against $S$. sonnei as a comparatively conserved target relative to disease burden, and its comparability to subtypes of other Shigella spp.

Early global population structure studies revealed that each Shigella species is delineated into multiple WGSA subtypes ${ }^{22-25}$. Specifically, S. flexneri comprises seven phylogroups (PGs) ${ }^{22}$ and $S$. sonnei, five lineages ${ }^{26}$. To describe the genomic epidemiology of the GEMS Shigella within existing frameworks, we constructed species phylogenetic trees and integrated these with epidemiological metadata and publicly available genomes. The S. flexneri phylogeny revealed two distinct lineages separated by $\sim 34,000$ SNPs; one comprising five previously described $\mathrm{PGs}^{22}$ and a distant clade comprising largely $S$. flexneri serotype 6 isolates (herein termed Sf6), contributing distinctly to the disease burden of each country (Fig. 2 and Supplementary Fig. 3). Phylogenetic analysis of S. sonnei revealed that all but two isolates belonged to the globally dominant multidrug resistant (MDR) Lineage III $^{23}$ (Supplementary Fig. 4). For S. boydii and S. dysenteriae, a total of three and two previously described phylogenetic clades ${ }^{25,27}$ were identified, respectively (Supplementary Fig. 5). Marked phylogenetic association of isolates with country of origin prompted an examination of species genomic diversity by region (East Africa, West Africa and Asia) and revealed that while $S$. flexneri diversity was comparable across regions, diversity varied by region for the remaining species (Extended Data Fig. 3). Specifically, S. sonnei was more genomically diverse in East Africa owing to the presence of two Lineage II isolates from Mozambique. For S. boydii, Asia contained greater diversity than African regions, owing to isolates belonging to additional clades. S. dysenteriae diversity was lower in West Africa relative to other regions by virtue of having only one circulating clade. Except for S. sonnei, similar trends were also observed for regional Shigella serotype/subserotype diversity (Extended Data Fig. 4). These geographical differences highlight the importance of considering regional variations during vaccine development and that vaccine candidates should be evaluated across multiple regions.

One limitation of the GEMS dataset is its constraints in geographical regions and time (being sampled between 2007 and 2011). However, several pieces of evidence support the utility of GEMS as being representative of Shigella in time and space. Specifically, the prevalence and regional distribution of Shigella serotypes across African GEMS sites were similar to those observed in the replicate Vaccine Impact of Diarrhea in Africa (VIDA) study conducted between 2015 and $2018^{28}$. Furthermore, recent large-scale genomic analyses of $S$. sonnei revealed that isolates sampled from a broad range of South Asian countries belonged to the same genomic subtype as the majority of GEMS isolates ${ }^{26}$. Finally, publicly available data for S. flexneri from LMICs sampled until 2021 were phylogenetically admixed with GEMS isolates (see below and Extended Data Fig. 7). Thus, GEMS has ongoing relevance as being representative of the diversity of Shigella targeted for control.

\section{Genomic subgroups as an alternative targeting method}

As GEMS was a case-control study, the dataset comprised Shigella case isolates derived from patients with MSD and control isolates from children without diarrhoea ${ }^{2}$ (see Methods). To explore the utility of vaccination targeting genomic subtype (relative to targeting serotype) for $S$. flexneri, we determined the relative effect size of the dominant subtype on the epidemiological outcome of shigellosis (that is, isolates derived from case patients rather than from controls). The dominant genomic subtype was PG3, which comprised the majority $(47 \%, 378 / 806)$ of total isolates, as well as case $(50 \%, 341 / 687)$ isolates, with some regional variation (Fig. 2). This resulted in an increased odds ratio ofcase status $(\mathrm{OR}=2.3,95 \%$ $\mathrm{CI}=1.5-3.6, P=0.0001)$ for PG3 compared with other genomic subtypes (PGs and Sf6) (Methods and Supplementary Table 4). The association of cases with the dominant serotype, S. flexneri serotype 2 a (accounting for 29\% (234/806) of total isolates and $31 \%$ (210/687) of case isolates) also resulted in an increased odds ratio of case status $(\mathrm{OR}=1.9,95 \% \mathrm{CI}=1.7-3.2, P=0.0099)$ (Supplementary Table 4). However, the higher prevalence of cases and larger effect size on case status of PG3 relative to serotype 2 a offer compelling evidence that targeting vaccination by phylogroup might offer broader coverage per licenced vaccine relative to a serotype-specific approach. Hence, finding common surface-exposed antigens that are conserved within phylogroups causing the major burden of disease may be an effective vaccine design approach that can provide greater efficacy than serotype-targeted vaccines.

\section{Diversity of S. flexneri relevant to serotype-targeted vaccines}

The development of serotype-targeted vaccines is complicated by the diversity and distribution of serotypes, which are heterogenous over time and geography ${ }^{8,21,29,30}$. Furthermore, genetic determinants of $\mathrm{O}$-antigen modification are often encoded on mobile genetic elements ${ }^{31,32}$ that can move horizontally among and within bacterial populations, causing the recognized, but poorly quantified phenomenon of serotype switching ${ }^{22,30,31}$, and resulting in the rapid escape of infection-induced immunity against homologous serotypes. For our analyses of serotype switching, we focused on S. flexneri owing to its 
a

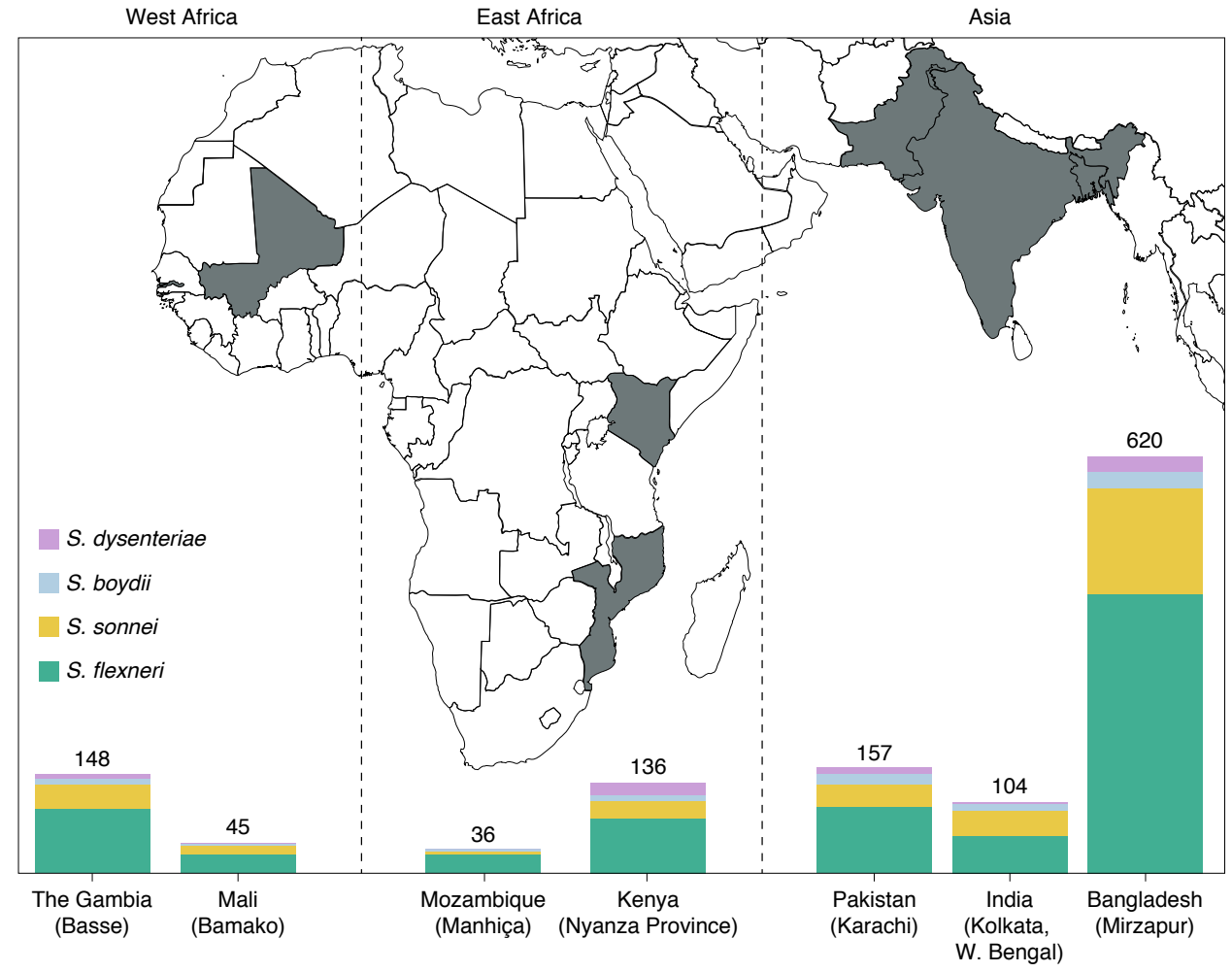

GEMS site

b

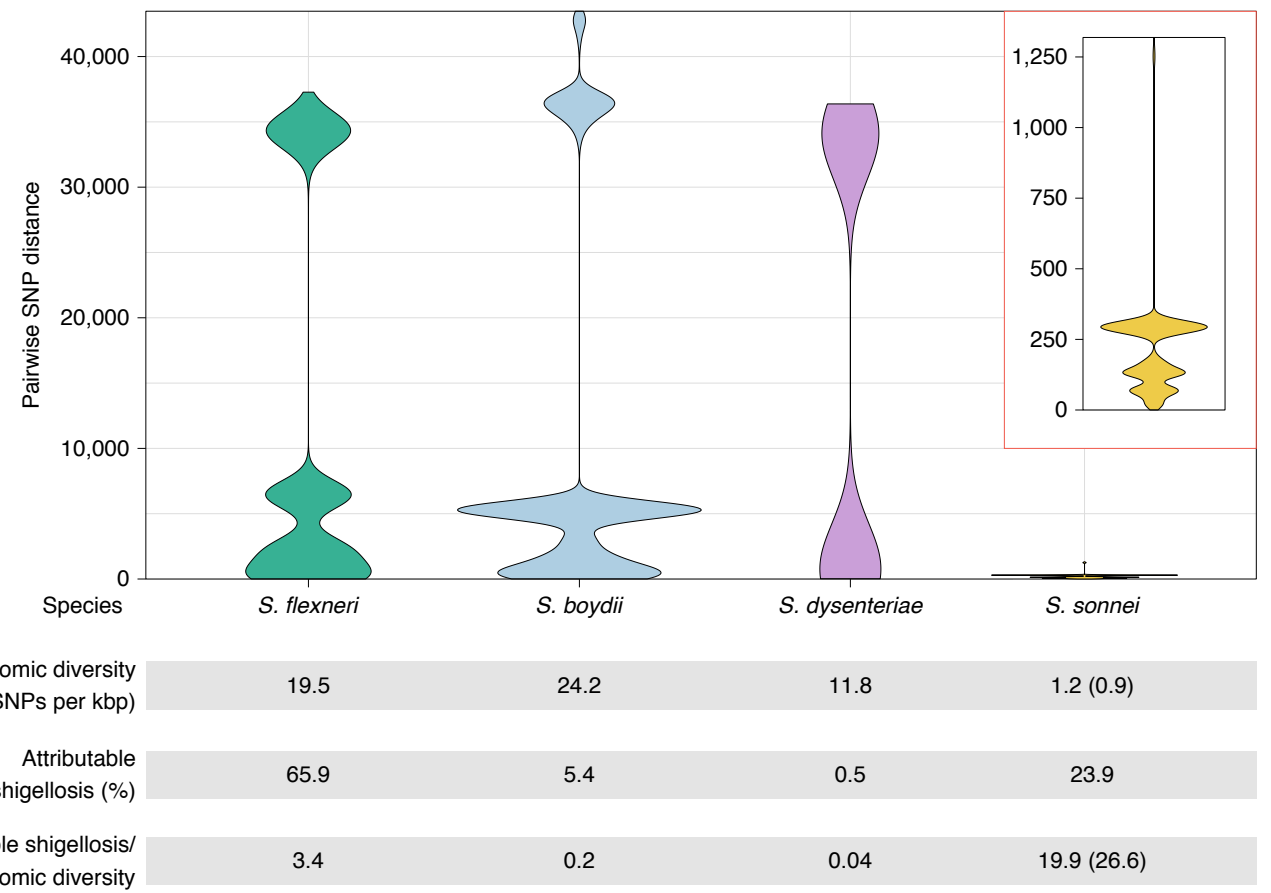

Fig. 1 | The diversity of Shigella spp. across seven LMICs. a, Stacked bar graphs illustrate the number of isolates from each Shigella spp. sequenced from GEMS and used in the current study, grouped by country. The seven countries from GEMS are highlighted in grey on the map and the selected field site(s) from each country are shown in brackets. b. Violin plots of pairwise genomic distances (in SNPs) among Shigella isolates within subgroups. Inset: a magnified plot for S. sonnei. The table below the plots shows the genomic diversity (as measured by the total number of SNPs per kbp (Methods)), the contribution to GEMS shigellosis burden and the shigellosis burden relative to genomic diversity for each species. For S. sonnei, the genomic diversity and shigellosis burden relative to genomic diversity calculated excluding the two outliers are shown in brackets.

high disease burden and serotypic diversity. Phenotypic serotyping data were overlaid onto the phylogeny and revealed that while there was a generally strong association of genotype (that is, PG/Sf6) with serotype (Fisher's exact test, $P<2.20 \times 10^{-16}$ ), multiple serotypes were observed for each genotype (Fig. 3). The greatest serotype diversity was observed in PG3, comprising seven distinct serotypes 


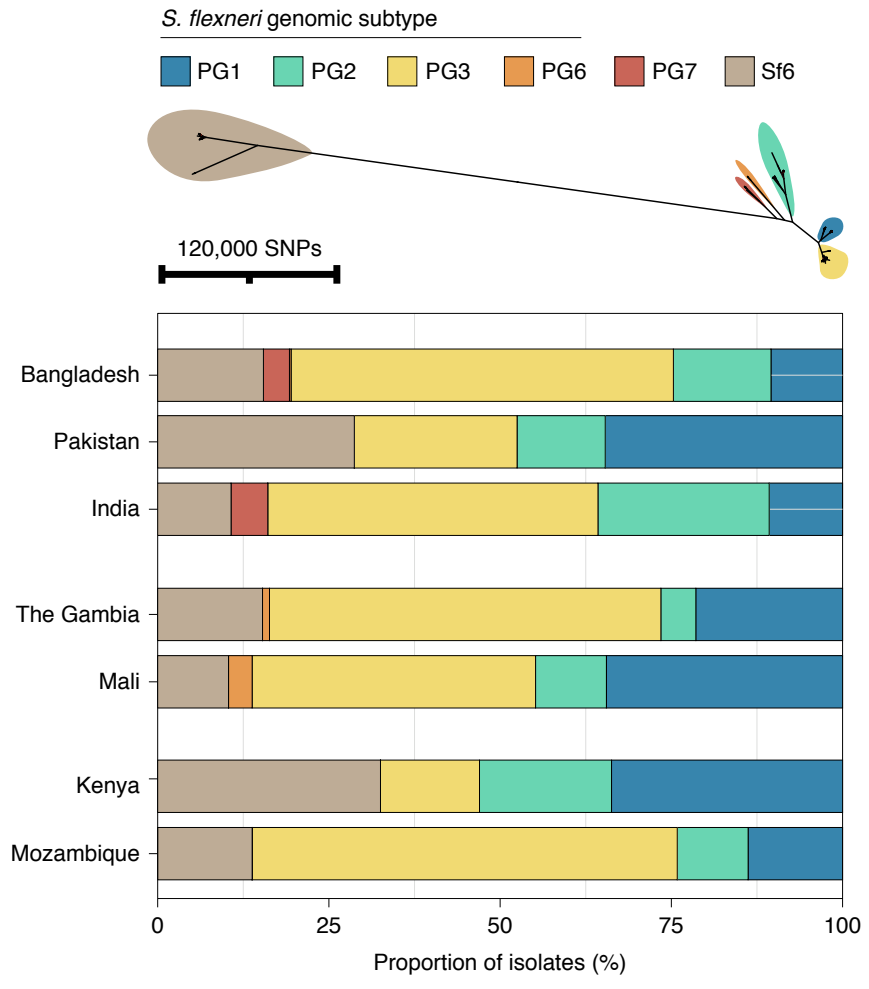

Fig. 2 | The diversity of S. flexneri genomic subtypes across seven GEMS study sites. Top: an unrooted maximum likelihood phylogenetic tree of $S$. flexneri genomes identified six distinct genomic subtypes, each highlighted in a different colour according to the key displayed above the tree. Bottom: barplot showing the relative frequencies of the subtypes at each GEMS site.

and two subserotypes. Correlation of serotypic diversity (number of serotypes) and genomic diversity (maximum pairwise SNP distance within genotype) revealed no evidence for an association (Extended Data Fig. 5). However, a significant positive correlation of serotypic diversity with the number of isolates in each genotype was found, indicating that serotype diversity scales with prevalence.

To qualitatively and quantitatively determine serotype switching across $S$. flexneri, we examined the number of switches occurring within each genotype. A switching event was inferred when a serotype emerged (either as a singleton or monophyletic clade) that was distinct from the majority (>65\%) serotype within a genotype (Fig. 3 and Extended Data Fig. 6). PG6 was excluded from the analysis, as only three isolates from GEMS belonged to this genotype and a dominant serotype could not be inferred. Quantitatively, this revealed that serotype switching was infrequent, with only 26 independent switches (3.3\% of isolates) identified across the five S. flexneri genotypes. Although the frequency of switching varied across the genotypes, statistical support for an association of serotype switching with genotype fell short of significance (Fisher's exact test, $P=0.09)$. Qualitatively, the majority $(22 / 26)$ of switching resulted in a change of serotype, with few (4/26) resulting in a change of subserotype. Examination of $\mathrm{O}$-antigen modification genes revealed that serotype switching was facilitated by changes in the presence or absence of various phage-encoded gtr and oac genes in the genomes, as well as point mutations in these genes (Supplementary Table 5). Our data also revealed that few (4/26) switching events resulted in more than two descendant isolates (Extended Data Fig. 6). This indicates that while natural immunity drives the fixation of relatively few serotype-switched variants in the short term, the potential pool of variants that could be driven to fixation by vaccine-induced

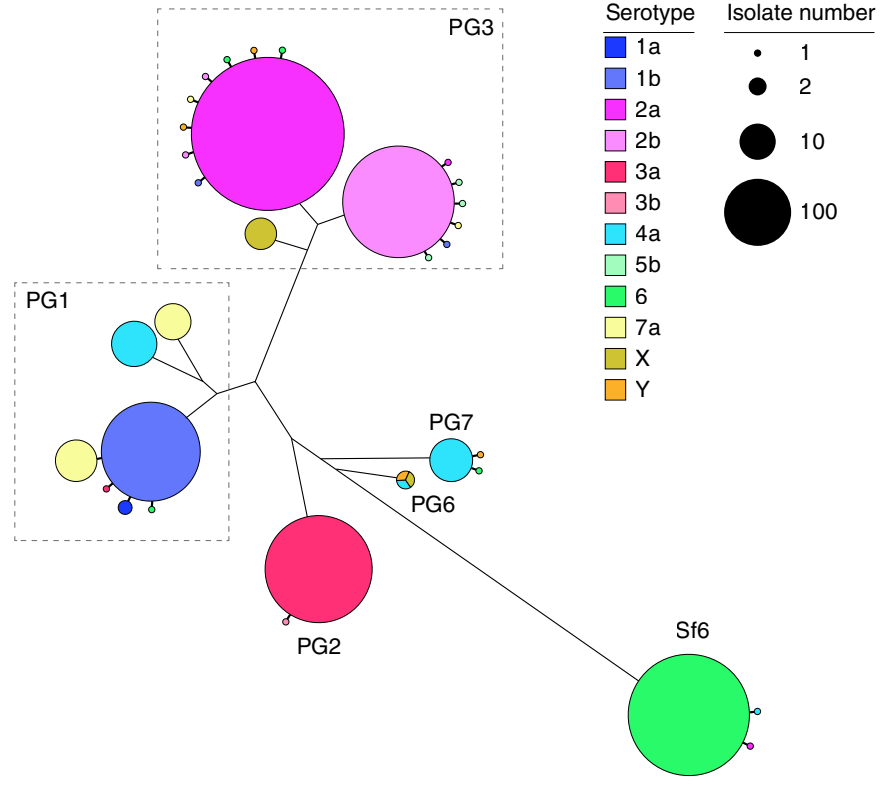

Fig. 3 | Diversity of S. flexneri population with respect to serotype switching. The unrooted S. flexneri phylogenetic tree is shown with the five phylogroups (PG1-PG7) and Sf6 labelled accordingly. For each genomic subtype, monophyletic clusters of the dominant serotype are shown collapsed into bubbles coloured according to the inlaid key. Single isolate or groups of isolates within a subtype of an alternative serotype are represented by further branches, indicating a single serotype switch.

The dashed rectangles group together multiple serotypic clusters belonging to the same PG. Bubble size indicates the number of isolates within a single cluster.

selective pressure following a serotype-targeted vaccination programme is much larger.

To estimate the probable timeframe over which serotype switching events might be expected to occur, we estimated the divergence time of the phylogenetic branch giving rise to each switching event. To streamline the analysis, we focused on two subclades of PG3, the most prevalent phylogroup, in which seven independent serotype switching events were detected (Supplementary Fig. 6). On the basis of the timeframes observed within our sample (spanning 4 years from 2007 to 2010), serotype switching was estimated to occur within an average of $348 \mathrm{~d}$, ranging from $159 \mathrm{~d}$ (95\% highest posterior density (HPD): $16-344)$ to $10,206 \mathrm{~d}$ (28 years) $(95 \%$ HPD: 5,494-15,408) (Supplementary Table 6). Taken together, our data show that although serotype-switching frequency is low, it can occur over relatively short timeframes and lead to serotype replacement such that non-vaccine serotypes could replace vaccine serotypes following a vaccination programme, as has been observed for Streptococcus pneumoniae $e^{33,34}$. Consequently, serotype switching may impact the long-term effectiveness of vaccines that only provide serotype-specific protection against $\mathrm{O}$-antigens. This highlights the advantages of protein-based or multivalent component approaches, such as the Invaplex or live attenuated vaccines that target both carbohydrates and protein antigens $s^{6,35}$.

\section{Heterogeneity among Shigella vaccine protein antigens}

Although conserved antigen-targeted vaccines can overcome some hurdles of serotype-targeted vaccines, they are also subject to complications arising from genetic diversity. Hence, we performed detailed examination of six protein antigens that are currently in development and have demonstrated protection in animal models (Supplementary Table 1). First, we assessed the distribution of the 

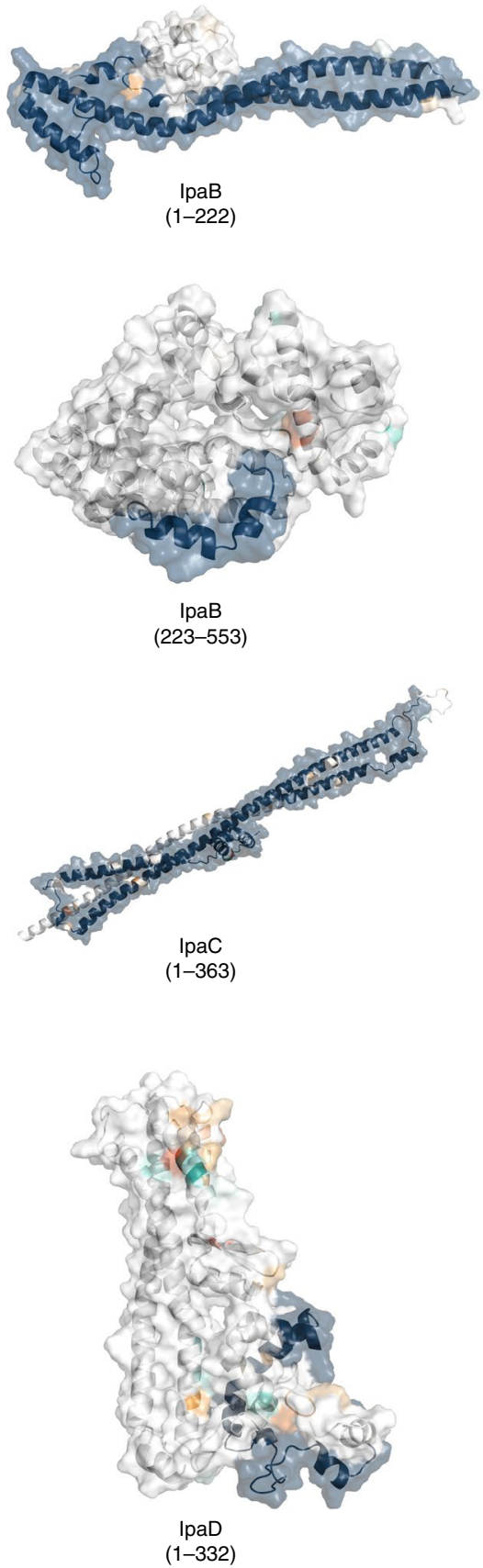
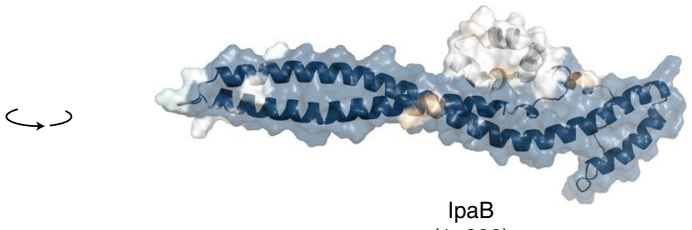

$(1-222)$

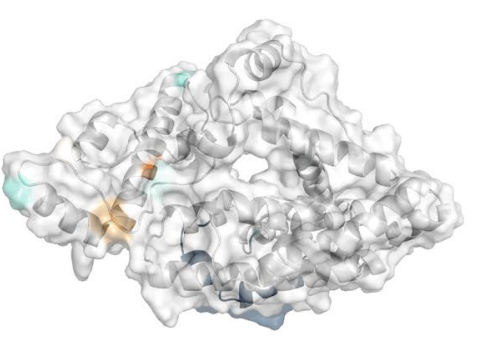

IpaB

$(223-553)$

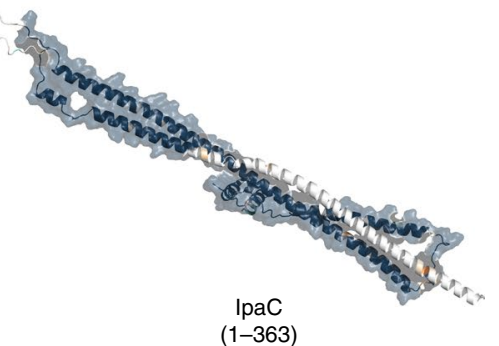

Epitopic region
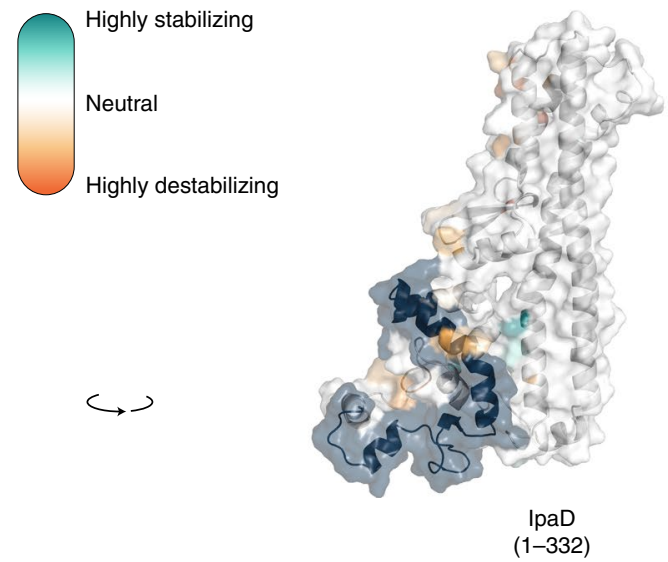

Fig. 4 | Visualization of mutations and its predicted effect on modelled IpaB, IpaC and IpaD protein antigens. Visualization of mutations on modelled proteins IpaB, I paC and IpaD. The protein residue ranges modelled are shown in brackets. Blue regions represent empirically determined epitopes. Mutations identified within the proteins are coloured using the scale shown in the inlaid key. Visualisations in the right hand column are 180-degree rotations of the models relative to the left hand column.

candidates among GEMS Shigella isolates, which revealed that the proportional presence of antigens varied across species and with genetic context (Supplementary Fig. 7a). Specifically, genes encoded on the virulence plasmid ( $i p a B, i p a C$, ipaD, ics $P$ ) were present in $>85 \%$ of genomes for each species, with the exception of $S$. son$n e i$. The low proportion $(\leq 5 \%)$ of virulence plasmid encoded genes detected among $S$. sonnei was caused by a similarly low detection of the virulence plasmid among S. sonnei (6\%) (Supplementary Fig. 7b), which probably arose due to loss during sub-culture ${ }^{36}$. In contrast, the chromosomally encoded ompA was present in $>98 \%$ of all isolates. While the sigA gene (carried on the chromosomally integrated SHI-1 pathogenicity island) was present in $99 \%$ of S. sonnei genomes, it was identified in only $63 \%$ of S. flexneri genomes. Notably, among $S$. flexneri genomes, the sigA gene was exclusively found in PG3 and Sf6, and was present in $>96 \%$ of isolates in each genotype (Supplementary Fig. 3), indicating an appropriate distribution for targeting the two genotypes. Second, we assessed the antigens for amino acid variation and modelled the probable impact of detected variants, since antigen variation may also lead to vaccine escape, as demonstrated for the P1 variant of SARS-CoV- $2^{37,38}$. We determined the distribution of pairwise amino acid (aa) sequence identities per antigen against $S$. flexneri vaccine strains for each species (see Methods). Overall, sequence identities were $>90 \%$, but varied with antigen (Supplementary Fig. 7a). For example, OmpA 


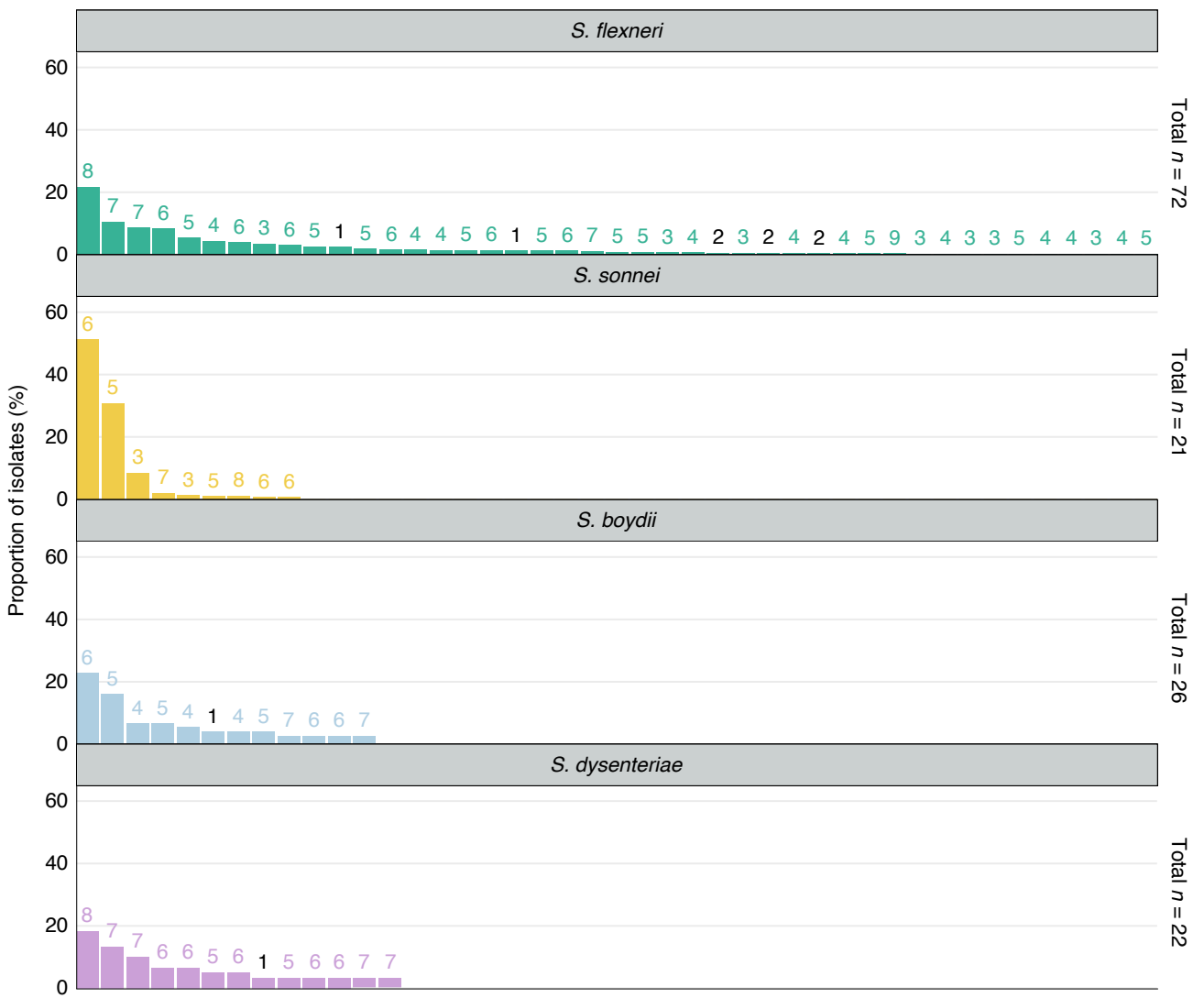

Fig. 5 | AMR genotypic profile diversity among Shigella spp. Each barplot represents the frequencies of AMR genotypic profiles among individual Shigella species. The number of bars shown along the $x$ axis represents the number of unique genotypic AMR profiles detected in each species and plotted against the proportion of isolates belonging to each profile ( $y$ axis). The numbers above each profile indicate the number of antimicrobial classes impacted by the genotype. Profiles that impact only one or two antimicrobial classes (that is, are not MDR) are highlighted in black. AMR profiles identified in only a single isolate were not plotted and are displayed in Extended Data Fig. 9. The total number of profiles detected within each species are displayed on the right-hand side of each plot.

was present in the highest proportion of genomes, but showed $\sim 5 \%$ sequence divergence, while SigA was present in fewer genomes, but exhibited little divergence $(<0.5 \%)$ among species. The least conserved sequence was IpaD, ranging from 3 to $7 \%$ divergence within species.

Not all antigenic variation will affect antibody binding, so we performed in silico analyses of the detected variants to assess whether they may compromise the antigens as vaccine targets. Again, we focused our analyses on $S$. flexneri owing to its high disease burden and the probable complication of serotype-based vaccination strategies for this species. Furthermore, as Shigella vaccines are likely to be used broadly across LMICs, we expanded the analyses to include an additional 236 publicly available $S$. flexneri genomes (collected from 2007 and 2021, and sampled from various countries across Asia and Africa), which were phylogenetically admixed with the GEMS isolates (Extended Data Fig. 7). A total of 148 aa variants were detected across the six antigens, 58 (39\%) of which were associated with genotype (that is, belonging to either PGs $1-5$ or Sf6). Among the total variants detected, only 15 (10\%) were unique to the publicly available genomes (Extended Data Fig. 7 and Supplementary Table 8), indicating that the GEMS dataset captured the majority of the diversity across LMICs. We then determined if aa variants were located in immunogenic regions (that is, epitope/peptide fragment) (Supplementary Fig. 8) and assessed their potential destabilization of protein structure through in silico protein modelling. For IpaB, IpaC and IpaD, the epitopes have been empirically determined ${ }^{39,40}$. The sequence and location of peptide fragments of
SigA, IcsP and OmpA used in vaccine development are available ${ }^{41,42}$. Variants located within the immunogenic regions were identified for all antigens relative to PG3 reference sequences (Methods and Fig. 4). Only 5 of 148 variants were predicted to be highly destabilizing to protein structure, and these occurred in: OmpA (residue 89 ) at a periplasmic turn, SigA (residues 1233 and 1271) in adjacent extracellular turns in the translocator domain (Supplementary Fig. 9), IcsP (residue 191) within the extracellular region of the beta barrel, and IpaD (residue 247) within a beta-turn-beta motif flanking the intramolecular coiled-coil (Fig. 4). None of the five destabilizing variants were located within the epitope/peptide region of the vaccine candidates.

While it remains possible that mutations could affect antigenicity through the disruption of folding or global stability, it is less likely than if they occurred in immunogenic regions. Our results thus indicate that it is less likely that existing natural variation will compromise antigen-based vaccine candidates for Shigella compared with serotype-based vaccines. However, any in silico approaches have limitations and functional immunological experiments will be required to determine the true impact of these variations on the antigen structure and its antigenicity. Furthermore, the knowledge base regarding the structure of antigens is currently incomplete. For example, there was no suitable template available for IpaC, and some epitopes were predicted to be in membrane regions which should be inaccessible to antibodies, indicating the need for more accurate publicly available protein structures to be developed for many of the vaccine antigen candidates. Finally, $90 \%$ of the antigenic variants 


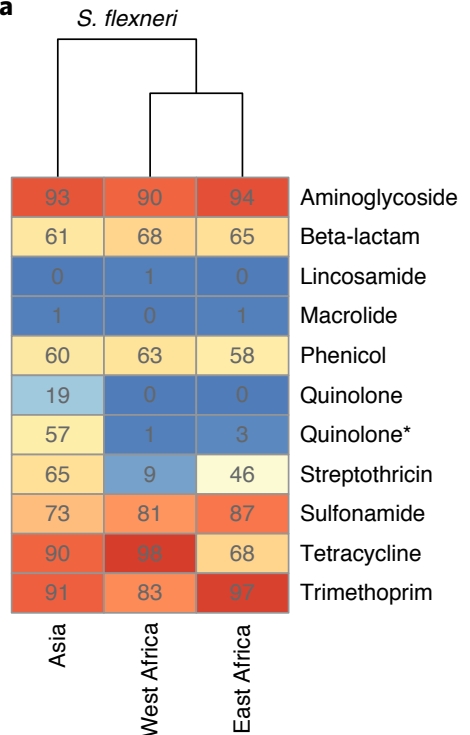

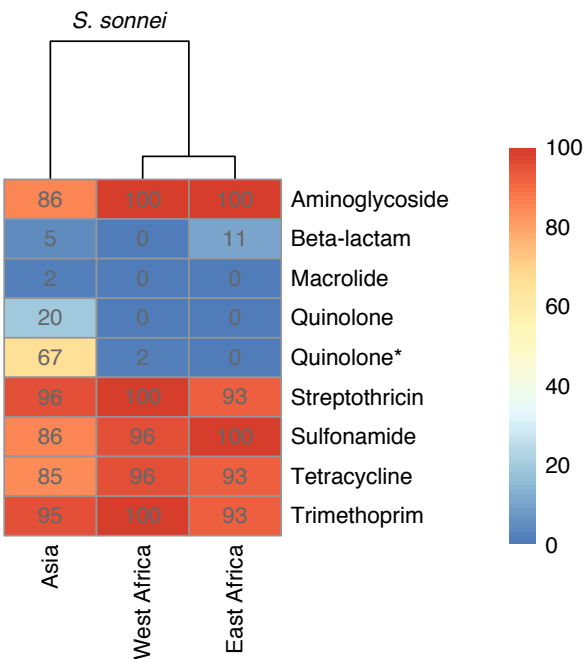

b
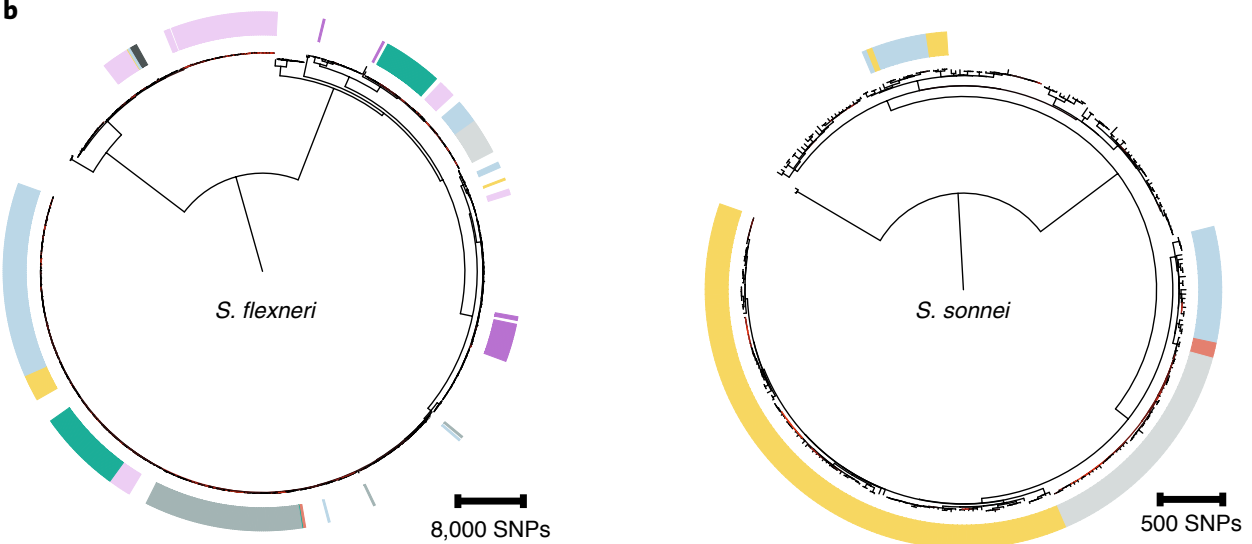

QRDR mutations

$\square$ Single (gyrA S83A)
$\square$ Single (gyrA S83L)
$\square$ Single (gyrA D87G)
$\square$ Single (gyrA D87N)
$\square$ Single (gyrA D87Y)

Double (gryA S83L parC S80I)

Triple (gyrA S83L gyrA D87G parC S80I)

Triple (gyrA S83L gyrA D87N parC S80I)

Triple (gyrA S83L gyrA D87Y parC E84K)

Fig. 6 | AMR genotypes grouped by region and convergent evolution of ciprofloxacin resistance. a, Detection of known AMR genetic determinants associated with drug class grouped by region. Each cell in the heatmap represents the percentage of isolates from a region containing genetic determinants associated with resistance to a drug class. Genetic determinant conferring reduced susceptibility to quinolone is indicated with an asterisk. b. The genetic convergent evolution of ciprofloxacin resistance in S. flexneri and S. sonnei. The presence of multiple monophyletic clades of QRDR mutations (single, double or triple according to the inlaid key) conferring reduced susceptibility or resistance to ciprofloxacin is shown in the outer ring. Figures for S. boydii and S. dysenteriae are shown in Supplementary Fig. 10.

were captured by GEMS, further supporting the representativeness of this dataset across time and space. Nevertheless, the presence of an additional destabilizing mutation in the more recent publicly available data highlights the need for ongoing surveillance across LMICs.

\section{Region-specific details of antimicrobial resistance}

Until a licenced vaccine is available, we must continue to treat shigellosis with supportive care and antimicrobials, for which the current WHO recommendation is the fluoroquinolone, ciprofloxa$\mathrm{cin}^{43}$. However, FQR Shigella is currently on the rise and spreading globally ${ }^{44}$. To examine AMR prevalence among GEMS isolates for evaluating treatment recommendations, we screened for known genetic determinants (horizontally acquired genes and point mutations) conferring resistance or reduced susceptibility to antimicrobials. Although we used only minimal phenotypic data, phenotypic resistance and genotypic prediction correlate well in S. flexneri and $S$. sonnei ${ }^{45,46}$. Our analysis revealed that $95 \%(1,189 / 1,246)$ of isolates were multidrug resistant (MDR), carrying AMR determinants against three or more antimicrobial classes (Fig. 5). S. flexneri exhibited the greatest diversity of AMR determinants, with a total of 45 identified determinants across the population, comprising 38 AMR genes and 7 point mutations (Extended Data Fig. 8 and Supplementary Table 2), and an extensive AMR genotype diversity of 72 unique resistance profiles (Fig. 5 and Extended Data Fig. 9). In contrast, $S$. sonnei exhibited the least diversity, with only 23 AMR 
determinants and 21 unique resistance profiles. An intermediate and comparable degree of AMR diversity was observed for both S. dysenteriae and S. boydii.

Overall, a high frequency of AMR genes conferring resistance against aminoglycoside, tetracycline, trimethoprim and sulfonamide antimicrobials was observed, while resistance against other antimicrobial classes varied with region and species (Fig. 6a and Supplementary Fig. 10a). The extended spectrum beta-lactamase gene blaCTX-M-15 was detected in a small $(9 / 1,246)$ percentage of isolates, and genes conferring resistance to macrolides and lincosamides were also infrequent (Extended Data Fig. 8), indicating that the recommended second-line treatments probably remain effective antimicrobials ${ }^{47}$.

However, higher rates of resistance were found against the first-line treatment. FQR in Shigella can be conferred through the acquisition of FQR genes or, more typically, by point mutations in the chromosomal Quinolone Resistance Determining Region (QRDR) within the DNA gyrase (gryA) and the topoisomerase IV ( parC) genes. Single and double QRDR mutations are known to confer reduced susceptibility to ciprofloxacin and are evolutionary intermediates on the path to resistance, conferred by triple mutations in this region ${ }^{45,48}$. Overall, FQR genes were uncommon in S. flexneri $(4 \%, 33 / 806)$, S. sonnei $(1 \%, 3 / 305)$ and S. dysenteriae $(7 \%, 4 / 60)$, but were present in $32 \%(24 / 75)$ of $S$. boydii. QRDR mutations were identified in all species (Extended Data Fig. 8), but were more common among $S$. sonnei $(65 \%, 199 / 305)$ and S. flexneri $(54 \%, 435 / 806)$ than among S. boydii $(15 \%, 11 / 75)$ and $S . d y s e n t e-$ riae $(30 \%, 18 / 60)$. Among these, triple QRDR mutations were identified in 13\% (106/806) of S. flexneri and 14\% (44/305) of S. sonnei. Analysis of the QRDR mutants across the phylogenies indicates marked convergent evolution towards resistance across the genus. Specifically, all triple QRDR mutant S. sonnei belonged to one monophyletic subtype (previously described as globally emerging from Southeast $\mathrm{Asia}^{49}$ ), while three distinct triple QRDR mutational profiles were found across three polyphyletic $S$. flexneri genotypes (Fig. 6b). Thus, the polyphyletic distribution of single, double and triple QRDR mutants indicates continued convergent evolution of lineages with reduced susceptibility or increased resistance to FQR.

We then stratified the dataset by geographic region, which revealed that FQR was largely associated with isolates from Asia where fluoroquinolones are more frequently used compared with African sites (Fig. 6a and Supplementary Fig. 10a) ${ }^{50}$; this is consistent with trends observed in atypical enteropathogenic Escherichia coli isolated from GEMS ${ }^{50}$. Furthermore, analysis of African Shigella isolates from VIDA collected between 2015 and 2018 revealed that all species across West Africa and East Africa remained susceptible to ciprofloxacin ${ }^{28}$. Our analyses thus suggest that for the period of the GEMS trial (2007-2011), 17\% (150/881) of Shigella isolates from Asia were resistant and 58\% (508/881) had reduced susceptibility to the WHO-recommended antimicrobial. The high level of reduced susceptibility, together with marked convergent evolution towards resistance, suggests that management of shigellosis with fluroquinolones at these sites may soon be ineffective and regional antimicrobial treatment guidelines may require updating. These results indicate the value of AMR and genomic surveillance in LMICs for the control and management of shigellosis, and will be improved by initiatives such as the Africa Pathogen Genomics Initiative ${ }^{51}$ and the WHO Global Antimicrobial Resistance Surveillance System ${ }^{52}$.

\section{Conclusions}

Pathogen genomics is a powerful tool that has a wide range of applications to help combat infectious diseases. Here we have applied this tool to an unparalleled systematically collected Shigella dataset to characterize the relevant population diversity of this pathogen across LMICs in a pre-vaccine era. This study has highlighted the urgent need to continue the development of Shigella vaccines for children in endemic areas. The genomic diversity in Shigella presents a major hurdle in controlling the disease and we have demonstrated the anticipated pitfalls of current vaccination approaches, emphasizing the importance of considering the local and global diversity of the pathogens in vaccine design and implementation. The relatively low heterogeneity among protein vaccine antigens in the S. flexneri population, and the lack of mutations predicted to be destabilizing, support the use of conserved antigens, and/or their inclusion alongside serotype-specific approaches for improved vaccine design. Our results also revealed that current antimicrobial treatment guidelines for shigellosis should be updated, particularly in Asia, and that improved and ongoing surveillance is essential to guide antimicrobial stewardship. Taken together, this study demonstrates the benefit of genomics in guiding prevention and control of shigellosis, providing further impetus to continue working to overcome the challenges associated with the implementation of WGS for pathogen surveillance in LMICs. Finally, our results suggest that annual Shigella surveillance would probably identify serotype switching, which would be especially important following the introduction of a vaccination programme. Although our results are focused on shigellosis, our approach is translatable to other bacterial pathogens and is particularly relevant as we enter the era of vaccines for AMR.

\section{Methods}

Dataset, bacterial isolates and sequencing. A total of 1,264 Shigella isolates from both cases and controls collected during GEMS were investigated in this study ${ }^{2,3}$. According to the GEMS study design, case enrolment required each child with diarrhoea (diarrhoea was defined as three or more loose stools within the previous $24 \mathrm{~h}$ ) seeking care at a selected sentinel hospital or health centre to fulfill at least one of the criteria for $\mathrm{MSD}^{2}$. Controls were enrolled as children without diarrhoea, matched to every individual patient with MSD by age, sex and residential area. All isolates were derived from stool samples/rectal swabs: their identification, confirmation and isolation have been described previously ${ }^{21}$ A total of 1,344 isolates were sequenced at the Earlham Institute, with genomic DNA extraction, sequencing library construction and whole-genome sequencing carried out according to the Low Input Transposase Enabled (LITE) pipeline ${ }^{53}$. Among these, 225 isolates failed quality controls having mean sample depths of coverage $<10 \times$ and an assembly sizes of $<4 \mathrm{MB}$ and were re-sequenced. For these isolates, genomic DNA was re-extracted at the University of Maryland School of Medicine (Baltimore, MD) from cultures grown in Lysogeny Broth overnight. DNA was extracted in 96-well format from $100 \mu \mathrm{l}$ of sample using the MagAttract PowerMicrobiome DNA/RNA Kit (Qiagen) automated on a Hamilton Microlab STAR robotic platform. Bead disruption was conducted on a TissueLyser II $(20 \mathrm{~Hz}$ for $20 \mathrm{~min})$ instrument in a 96 -well deep-well plate in the presence of $200 \mu \mathrm{l} \mathrm{phenol/chloroform.} \mathrm{Genomic} \mathrm{DNA} \mathrm{was} \mathrm{eluted} \mathrm{in} 90 \mu \mathrm{l}$ water after magnetic bead clean up and the resulting genomic DNA was quantified by Pico Green. The genomic DNA was shipped to the Centre for Genomic Research (CGR, University of Liverpool) for whole-genome sequencing. Sequencing library was constructed using NEBNext Ultra II FS DNA Library Prep Kit for Illumina and sequenced on the Illumina NovaSeq 6000 platform, generating $150 \mathrm{bp}$ paired-end reads.

An additional 125 publicly available Shigella and E. coli reference genomes were included in the phylogenetic analyses and a further 236 S. flexneri genomes were included in the assessment of vaccine protein antigens. Details of GEMS and reference genomes analysed in this study are listed in Supplementary Tables 2 and 3 , respectively.

Sequence mapping and variant calling. Adaptors and low-quality bases were trimmed with Trimmomatic v0.38 ${ }^{54}$, and reads qualities were assessed using FastQC v0.11.6 (https://www.bioinformatics.babraham.ac.uk/projects/fastqc/) and MultiQC v1.7 $7^{55}$. Filtered reads were mapped against Shigella reference genomes with BWA mem v0.7.17 $7^{56}$ using default parameters. S. flexneri, S. sonnei, S. boydii and $S$. dysenteriae sequencing reads were mapped against reference genomes from Sf2a strain 301 (accession NC_004337), Ss046 (accession NC_007384), Sb strain CDC 3083-94 (accession NC_010658) and Sd197 (accession NC_007606), respectively. Mappings were filtered and sorted using the SAMtools suite v1.9-47 ${ }^{57}$, and optical duplicate reads were marked using Picard v2.21.1-SNAPSHOT MarkDuplicates (http://broadinstitute.github.io/picard/). QualiMap v2.2.2 ${ }^{58}$ was used to evaluate mapping qualities and estimate mean sample depth of coverage. Sequencing reads for isolates sequenced using the LITE pipeline and re-sequenced at the CGR were combined to increase overall sample depth of coverage. Sequence variants were identified against reference using SAMtools v1.9-47 mpileup and bcftools v1.9-80 $0^{57}$. Low-quality SNPs were filtered if mapping quality was $<60$, Phred-scaled quality score was $<30$ and read depth was $<4$. 
Phylogenetic reconstruction, inference of genomic diversity, and genotyping. Filtered SNP variants were used to generate a reference-based pseudogenome for each sample, where regions with depth of coverage $>4 \times$ were masked in the pseudogenome. Additionally, regions containing phage (identified using PHASTER $^{59}$ web server) and insertion sequences were identified from the reference genomes, and co-ordinates were used to mask these sites on the pseudogenomes using BEDTools v2.28.0 maskfasta ${ }^{60}$. For each species, chromosome sequences from the masked pseudogenomes were extracted and concatenated. Gubbins v2.3.4 ${ }^{61}$ was used to remove regions of recombination and invariant sites from the concatenated pseudogenomes (Supplementary Fig. 4). This generated a chromosomal SNP alignment length of 78,251 bp for $S$. flexneri $(n=806)$, $5,081 \mathrm{bp}$ for $S$. sonnei $(n=305), 98,842 \mathrm{bp}$ for $S$. boydii $(n=75)$ and $45,031 \mathrm{bp}$ for $S$. dysenteriae $(n=60)$. Maximum-likelihood phylogenetic reconstruction was performed independently for each species and inferred with IQ-TREE v2.0-rc2 ${ }^{62}$ using the FreeRate nucleotide substitution, invariable site and ascertainment bias correction model, with 1,000 bootstrap replicates. To contextualize GEMS isolates within the established genomic subtypes and to infer the most appropriate root for each species tree, phylogenetic trees were reconstructed including publicly available reference genomes of isolates from previously defined lineages/ phylogroups/clades and E. coli isolates (Supplementary Table 3). The phylogenetic trees for S. flexneri, S. boydii and S. dysenteriae waere rooted using E. coli strain IAI1-117 (accession SRR2169557) as an outgroup. The phylogenetic tree for S. sonnei was midpoint rooted. Visualizations were performed using interactive Tree of Life (iTOL) v6.1.1 ${ }^{63}$. Assignment of S. sonnei genomes to hierarchical genotypes was performed using the script sonnei_genotype.py (https://github.com/ katholt/sonneityping) on the basis of mapping files, and according to a previously described genotyping scheme ${ }^{26}$

To measure the extent of Shigella genomic diversity among the GEMS population, pairwise SNP distance was determined from the alignment of core genome SNPs identified outside regions of recombination using snp-dists v0.7.0 (https://github.com/tseemann/snp-dists). For each species, the genomic diversity, measured by SNPs per kbp, was determined by dividing the core genome SNP alignment length by the core genome size (S. flexneri $4,015,307 \mathrm{bp}, S$. sonnei $4,177,070 \mathrm{bp}, \mathrm{S}$. boydii 4,088,693 bp and S. dysenteriae 3,821,602 bp). To scale the proportion of disease burden attributable to the genome diversity of each species, the percentage of species contribution to GEMS shigellosis disease burden was divided by the number of SNPs per kbp.

Serotype switching timeframe inference. To estimate the probable timeframe of serotype switching, we performed temporal phylogenetic reconstruction to infer the time of divergence along branches exhibiting serotype switching. We streamlined the analysis and focused on isolates belonging to two subclades of S. flexneri PG3. First, for each of the two subclades $(n=99$ and $n=45)$, a maximum-likelihood phylogeny was reconstructed on the basis of genome multiple sequence alignments (described above). Then, TempEst v1.5.3 ${ }^{64}$ was used to determine whether there was sufficient temporal signal in the data by inferring linear relationship between root-to-tip distances of the phylogenetic branches and the year of sample isolation. Data from both subclades revealed positive correlation between sampling time and phylogenetic root-to-tip divergence, with $R^{2}$ of 0.186 and 0.111 (Extended Data Fig. 10). Once temporal signals within each of the two datasets were confirmed, core genome SNP alignments of length $559 \mathrm{bp}$ and $1,244 \mathrm{bp}$ were analysed independently using BEAST2 v2.6.1 ${ }^{65}$. The parameters were as follows: dates specified as days, bModelTest ${ }^{66}$ implemented in BEAST2 was used to infer the most appropriate substitution model, a relaxed log normal clock rate with a coalescent Bayesian skyline model for population growth. Beauti v2.6.3 ${ }^{65}$ was used to general xml configuration files. A total of five independent chains were performed, each with chain length of 250,000,000, logging every 1,000 and accounting for invariant sites. Convergence of each run was visually assessed with Tracer v1.7.167, with all parameter effective sampling sizes $\geq 200$. Tree files were sampled and combined using LogCombiner v2.6.1, the combined files were then summarized using TreeAnnotator v2.6.0 with $10 \%$ burn-in to generate the Maximum Clade Credibility tree ${ }^{68}$. Divergence time was inferred by reading the branch length from the most recent common ancestor to the first sampled isolate that serotype-switched.

Genome assembly and annotation. Draft genome sequences were assembled using Unicycler v0.4. $7^{69}$ with -min_fasta_length set to 200 . QUAST v5.0.2 $2^{70}$ was used to assess the qualities of the assemblies. Assemblies with total assembly length outside the range of $<4 \mathrm{Mbp}$ and $>6.4 \mathrm{Mbp}$ were removed, resulting in an average length of 4,275,508 bp (range 4,004,109-4,538,734bp) for S. flexneri, 4,264,097 bp (range 4,008,630-4,779,279bp) for S. sonnei, 4,227,671 bp (range 4,000,714$4,689,815 \mathrm{bp}$ ) for S. boydii and 4,297,921 bp (range 4,040,642-4,659,860 bp) for $S$. dysenteriae. An average N50 value of 29,804 bp (range $6,810-34,658 \mathrm{bp}$ ) was generated for S. flexneri, 23,961 bp (range 11,547-30,008 bp) for S. sonnei, 20,835 bp (range 15,323-40,119bp) for $S$. boydii and 22,137 bp (range 14,090-31,358bp) for S. dysenteriae. Draft genomes were annotated using Prokka v1.13.3 ${ }^{71}$.

Pangenome analysis. The pangenome of each species was defined using Roary v3.12.0 $0^{72}$ without splitting paralogues. The pangenome accumulation curves were generated separately for each species using the specaccum function from Vegan v2.5-7 (https://github.com/vegandevs/vegan/), with 100 permutations and random subsampling. Inspections of the variable gene content showed that all four species had open pangenomes, implying that the number of unique genes increases with the addition of newly sequenced genomes.

Shigella flexneri molecular serotyping. Shigella serotype data were provided by collaborators at the University of Maryland School of Medicine; serotyping was performed as previously described ${ }^{21}$. In silico serotyping of S. flexneri genomes was performed using ShigaTyper v1.0.6 ${ }^{73}$, which detects the presence of serotype-determining genetic elements from sequencing reads to predict serotype. ShigaTyper predictions were $84 \%$ concordant with the serotype data provided. SRST2 $\mathrm{v}^{74}$ was used to detect mutations within serotype-determining genetic elements, and was run against ShigaTyper sequence database with default parameters.

Protein antigen screening. To determine the presence of antigen vaccine candidates among GEMS Shigella isolates, genes of the antigen vaccine candidates were screened against draft genome assemblies using screen_assembly ${ }^{19}$, with a threshold of $\geq 80 \%$ identity and $\geq 70 \%$ coverage to the reference sequence. Reference sequences for $i p a B$, ipaC, $i p a D$ and $i c s P$ were derived from $S$. flexner $i$ 5a strain M90T (accession GCA_004799585) and those for ompA and sigA were derived from S. flexneri 2a strain 2457 T (accession NC_004741), both strains being commonly used in the laboratory for vaccine development. Antigen sequence variations were determined by examining the BLASTp ${ }^{75}$ percentage identity against relevant query reference sequences. Allelic variations of antigen vaccine candidates among the $S$. flexneri population were identified manually by visualizing amino acid sequence alignments using AliView v1.26 ${ }^{76}$. Publicly available $S$. flexneri genomes were also integrated into the analysis, with assembled genomes downloaded from EnteroBase (accessed 25 August 2021), including all isolates sampled between 2007 and 2021 from across LMICs (Asia $n=155$ and Africa $n=81$ ). No samples from Latin America met these criteria.

Protein antigen modelling. To assess the effect of point mutations on protein stability and vaccine escape, six antigen candidates from $S$. flexneri PG3 were modelled: OmpA, SigA, IcsP, IpaB, IpaC and IpaD (Supplementary Table 1). PG3 was selected as it is the most prevalent phylogroup and is therefore the target of current vaccine development. To model the antigen targets, we first searched for a suitable template using HHPred ${ }^{77,78}$. Five of the six proteins (OmpA, SigA, IcsP, IpaB and IpaD) had suitable homologues available. To improve the performance of the comparative modelling, the signal peptides for OmpA, SigA and IcsP were removed and OmpA, SigA and IpaB were modelled in two parts to make use of optimal templates. RosettaCM source release- $188^{79}$ was used to generate 200 models for each of the five proteins using the single best available template. For IpaC, where no suitable templates were available, trRosetta ${ }^{80}$ was used to create five de novo predicted models. The best model for each antigen candidate was selected using QMEAN's v4.2.0 average local score. QMEANbrane v4.2.0 $0^{81,82}$ was used for suitable membrane proteins (IpaB, IpaC and IpaD), otherwise QMEANDisCo v4.2.0 ${ }^{81}$ was used (Supplementary Table 7). Full details of the modelling and ranking are shown in Supplementary Table 8. The effect of point mutations on the stability of the antigen candidates was assessed using PremPS ${ }^{83}$, and the default criterion of $\Delta \Delta \mathrm{G}>1 \mathrm{kcal} \mathrm{mol}^{-1}$ was used to define highly destabilizing mutations.

Detection of AMR genetic determinants and AMR testing. To detect the presence of known genetic determinants for AMR, AMRFinderPlus v3.9.3 $3^{84}$ was used to screen draft genome assemblies against the AMRFinderPlus database, which is derived from the Pathogen Detection Reference Gene Catalog (https:// www.ncbi.nlm.nih.gov/pathogens/). AMRFinderPlus was performed with the organism-specific option for Escherichia, to screen for both point mutations and genes, and filter out uninformative genes that were nearly universal in a group. The output was then filtered to remove genetic determinants identified with $\leq 80 \%$ coverage and $\leq 90 \%$ identity. The presence of $S$. sonnei virulence plasmid was confirmed using short-read mapping using BWA mem (as described above) against the reference virulence plasmid from Ss046 (GenBank accession CP000039.1). Presence of the plasmid was defined by the mapping of $>60 \%$ breadth of coverage across the reference. Visualizations of AMR resistance profiles were performed with UpSetR v2.1.3 ${ }^{85}$. Four $S$. flexneri isolates with triple QRDR mutations were phenotypically tested for ciprofloxacin resistance using the Kirby-Bauer standardized disk diffusion method ${ }^{86}$

Statistical analyses. The strength of association of $S$. flexneri genomic subtype and serotype with the occurrence of case outcome was calculated using MedCalc Software odds ratio calculator v20 (https://www.medcalc.org/calc/odds_ratio. php) to report the odds ratio, $95 \%$ confidence interval and statistical association. Association of genomic subtype with serotype and serotype switching was tested using Fisher's exact test. Linear regression analysis was used to determine the correlation between serotype diversity and various properties of genomic subtype. Both analyses were performed using R v4.0.3. 
Reporting Summary. Further information on research design is available in the Nature Research Reporting Summary linked to this article.

Reporting Summary. Further information on research design is available in the Nature Research Reporting Summary linked to this article.

\section{Data availability}

Short-read sequences supporting the findings of this study have been deposited in the European Nucleotide Archive (https://www.ebi.ac.uk/ena/) under the project accession number PRJEB45383. Accession numbers for isolates used in this study are listed in Supplementary Table 2. Publicly available sequences were downloaded from GenBank (https://www.ncbi.nlm.nih.gov/genbank/), Sequence Read Archive (https://www.ncbi.nlm.nih.gov/sra), the European Nucleotide Archive (https://www.ebi.ac.uk/ena) and EnteroBase (http://enterobase. warwick.ac.uk/). Accession numbers of publicly available genomes are listed in Supplementary Table 3. Phylogenetic trees, antigen protein models and BEAST input and output files have been deposited in FigShare (https://doi.org/10.6084/ $\mathrm{m} 9$.figshare.14743833). Source data are provided with this paper.

\section{Code availability}

All codes used in this study are described in the Methods. No custom algorithms were used for analyses.

Received: 14 June 2021; Accepted: 17 December 2021; Published online: 31 January 2022

\section{References}

1. Khalil, I. A. et al. Morbidity and mortality due to Shigella and enterotoxigenic Escherichia coli diarrhoea: the Global Burden of Disease Study 1990-2016. Lancet Infect. Dis. 18, 1229-1240 (2018).

2. Kotloff, K. L. et al. Burden and aetiology of diarrhoeal disease in infants and young children in developing countries (the Global Enteric Multicenter Study, GEMS): a prospective, case-control study. Lancet 382, 209-222 (2013).

3. Liu, J. et al. Use of quantitative molecular diagnostic methods to identify causes of diarrhoea in children: a reanalysis of the GEMS case-control study. Lancet 388, 1291-1301 (2016).

4. Kotloff, K. L., Riddle, M. S., Platts-Mills, J. A., Pavlinac, P. \& Zaidi, A. K. M. Shigellosis. Lancet 391, 801-812 (2018).

5. Shrivastava, S. R., Shrivastava, P. S. \& Ramasamy, J. World health organization releases global priority list of antibiotic-resistant bacteria to guide research, discovery, and development of new antibiotics. J. Med. Soc. 32 76 (2018).

6. Barry, E. M. et al. Progress and pitfalls in Shigella vaccine research. Nat. Rev. Gastroenterol. Hepatol. 10, 245-255 (2013)

7. Cohen, D., Green, M. S., Block, C., Slepon, R. \& Ofek, I. Prospective study of the association between serum antibodies to lipopolysaccharide $\mathrm{O}$ antigen and the attack rate of shigellosis. J. Clin. Microbiol. 29, 386-389 (1991).

8. Ferreccio, C. et al. Epidemiologic patterns of acute diarrhea and endemic Shigella infections in children in a poor periurban setting in Santiago, Chile. Am. J. Epidemiol. 134, 614-627 (1991).

9. Formal, S. B. et al. Effect of prior infection with virulent Shigella flexneri 2 a on the resistance of monkeys to subsequent infection with Shigella sonnei. J. Infect. Dis. 164, 533-537 (1991).

10. Kotloff, K. L. et al. A modified Shigella volunteer challenge model in which the inoculum is administered with bicarbonate buffer: clinical experience and implications for Shigella infectivity. Vaccine 13, 1488-1494 (1995).

11. Levine, M. M., Kotloff, K. L., Barry, E. M., Pasetti, M. F. \& Sztein, M. B. Clinical trials of Shigella vaccines: two steps forward and one step back on a long, hard road. Nat. Rev. Microbiol. 5, 540-553 (2007).

12. Mani, S., Wierzba, T. \& Walker, R. I. Status of vaccine research and development for Shigella. Vaccine 34, 2887-2894 (2016).

13. Talaat, K. R. et al. Human challenge study with a Shigella bioconjugate vaccine: analyses of clinical efficacy and correlate of protection. EBioMedicine 66, 103310 (2021).

14. Passwell, J. H. et al. Age-related efficacy of Shigella O-specific polysaccharide conjugates in 1-4-year-old Israeli children. Vaccine 28, 2231-2235 (2010).

15. Turbyfill, K. R., Kaminski, R. W. \& Oaks, E. V. Immunogenicity and efficacy of highly purified invasin complex vaccine from Shigella flexneri 2a. Vaccine 26, 1353-1364 (2008).

16. Martinez-Becerra, F. J. et al. Broadly protective Shigella vaccine based on type III secretion apparatus proteins. Infect. Immun. 80, 1222-1231 (2012).

17. Berlanda Scorza, F. et al. High yield production process for Shigella outer membrane particles. PLoS ONE 7, e35616 (2012).

18. Frenck, R. W. Jr. et al. Efficacy, safety, and immunogenicity of the Shigella sonnei 1790GAHB GMMA candidate vaccine: results from a phase $2 \mathrm{~b}$ randomized, placebo-controlled challenge study in adults. EClinicalMedicine 39, 101076 (2021).
19. Davies, M. R. et al. Atlas of group A streptococcal vaccine candidates compiled using large-scale comparative genomics. Nat. Genet. 51, 1035-1043 (2019).

20. Telford, J. L. Bacterial genome variability and its impact on vaccine design. Cell Host Microbe 3, 408-416 (2008).

21. Livio, S. et al. Shigella isolates from the global enteric multicenter study inform vaccine development. Clin. Infect. Dis. 59, 933-941 (2014).

22. Connor, T. R. et al. Species-wide whole genome sequencing reveals historical global spread and recent local persistence in Shigella flexneri. Elife 4, e07335 (2015)

23. Holt, K. E. et al. Shigella sonnei genome sequencing and phylogenetic analysis indicate recent global dissemination from Europe. Nat. Genet. 44, 1056-1059 (2012).

24. Njamkepo, E. et al. Global phylogeography and evolutionary history of Shigella dysenteriae type 1. Nat. Microbiol. 1, 16027 (2016).

25. Kania, D. A., Hazen, T. H., Hossain, A., Nataro, J. P. \& Rasko, D. A. Genome diversity of Shigella boydii. Pathog. Dis. 74, ftw027 (2016)

26. Hawkey, J. et al. Global population structure and genotyping framework for genomic surveillance of the major dysentery pathogen, Shigella sonnei. Nat. Commun. 12, 2684 (2021).

27. Sahl, J. W. et al. Defining the phylogenomics of Shigella species: a pathway to diagnostics. J. Clin. Microbiol. 53, 951-960 (2015).

28. Badji, H. et al. Prevalence, antimicrobial resistance, and distribution of Shigella among children under five in three sub-Saharan African countries in the Vaccine Impact on Diarrhea in Africa Study. in American Society of Tropical Medicine and Hygiene.

29. von Seidlein, L. et al. A multicentre study of Shigella diarrhoea in six Asian countries: disease burden, clinical manifestations, and microbiology. PLoS Med. 3, e353 (2006).

30. Ye, C. et al. Emergence of a new multidrug-resistant serotype $\mathrm{X}$ variant in an epidemic clone of Shigella flexneri. J. Clin. Microbiol. 48, 419-426 (2010).

31. Allison, G. E. \& Verma, N. K. Serotype-converting bacteriophages and O-antigen modification in Shigella flexneri. Trends Microbiol. 8, 17-23 (2000).

32. Sun, Q. et al. A novel plasmid-encoded serotype conversion mechanism through addition of phosphoethanolamine to the O-antigen of Shigella flexneri. PLoS ONE 7, e46095 (2012).

33. Weinberger, D. M., Malley, R. \& Lipsitch, M. Serotype replacement in disease after pneumococcal vaccination. Lancet 378, 1962-1973 (2011).

34. Brueggemann, A. B., Pai, R., Crook, D. W. \& Beall, B. Vaccine escape recombinants emerge after pneumococcal vaccination in the United States. PLoS Pathog. 3, e168 (2007).

35. Riddle, M. S. et al. Safety and immunogenicity of an intranasal Shigella flexneri 2a Invaplex 50 vaccine. Vaccine 29, 7009-7019 (2011).

36. McVicker, G. \& Tang, C. M. Deletion of toxin-antitoxin systems in the evolution of Shigella sonnei as a host-adapted pathogen. Nat. Microbiol. 2, 16204 (2016).

37. Garcia-Beltran, W. F. et al. Multiple SARS-CoV-2 variants escape neutralization by vaccine-induced humoral immunity. Cell 184, 2523 (2021).

38. Zhou, D. et al. Evidence of escape of SARS-CoV-2 variant B.1.351 from natural and vaccine-induced sera. Cell 184, 2348-2361 (2021).

39. Mills, J. A., Buysse, J. M. \& Oaks, E. V. Shigella flexneri invasion plasmid antigens B and C: epitope location and characterization with monoclonal antibodies. Infect. Immun. 56, 2933-2941 (1988).

40. Turbyfill, K. R., Mertz, J. A., Mallett, C. P. \& Oaks, E. V. Identification of epitope and surface-exposed domains of Shigella flexneri invasion plasmid antigen D (IpaD). Infect. Immun. 66, 1999-2006 (1998).

41. Czerkinsky, C. \& Kim, D. W. Shigella protein antigens and methods. US patent 8168203 (2012)

42. Pore, D., Mahata, N., Pal, A. \& Chakrabarti, M. K. Outer membrane protein A (OmpA) of Shigella flexneri 2a induces protective immune response in a mouse model. PLoS ONE 6, e22663 (2011).

43. Guidelines for the Control of Shigellosis, Including Epidemics Due to Shigella dysenteriae type 1 (WHO, 2005).

44. Chung The, H. \& Baker, S. Out of Asia: the independent rise and global spread of fluoroquinolone-resistant Shigella. Microb. Genom. 4, e000171 (2018).

45. Sadouki, Z. et al. Comparison of phenotypic and WGS-derived antimicrobial resistance profiles of Shigella sonnei isolated from cases of diarrhoeal disease in England and Wales, 2015. J. Antimicrob. Chemother. 72, 2496-2502 (2017).

46. Baker, K. S. et al. Intercontinental dissemination of azithromycin-resistant shigellosis through sexual transmission: a cross-sectional study. Lancet Infect. Dis. 15, 913-921 (2015).

47. Williams, P. C. M. \& Berkley, J. A. Guidelines for the treatment of dysentery (shigellosis): a systematic review of the evidence. Paediatr. Int. Child Health 38, S50-S65 (2018).

48. Chung The, H. et al. South Asia as a reservoir for the global spread of Ciprofloxacin-resistant Shigella sonnei: a cross-sectional study. PLoS Med. 13 e1002055 (2016) 
49. Chung The, H. et al. Dissecting the molecular evolution of fluoroquinolone-resistant Shigella sonnei. Nat. Commun. 10, 4828 (2019).

50. Ingle, D. J., Levine, M. M., Kotloff, K. L., Holt, K. E. \& Robins-Browne, R. M. Dynamics of antimicrobial resistance in intestinal Escherichia coli from children in community settings in South Asia and sub-Saharan Africa. Nat. Microbiol. 3, 1063-1073 (2018)

51. Makoni, M. Africa's \$100-million pathogen genomics initiative. Lancet Microbe 1, e318 (2020)

52. N.G.H.R.U.O.G.S.O., A. M. R. Whole-genome sequencing as part of national and international surveillance programmes for antimicrobial resistance: a roadmap. BMJ Glob. Health 5, e002244 (2020).

53. Perez-Sepulveda, B. M. et al. An accessible, efficient and global approach for the large-scale sequencing of bacterial genomes. Genome Biol. 22, 349 (2021)

54. Bolger, A. M., Lohse, M. \& Usadel, B. Trimmomatic: a flexible trimmer for Illumina sequence data. Bioinformatics 30, 2114-2120 (2014).

55. Ewels, P., Magnusson, M., Lundin, S. \& Kaller, M. MultiQC: summarize analysis results for multiple tools and samples in a single report. Bioinformatics 32, 3047-3048 (2016).

56. Li, H. Aligning sequence reads, clone sequences and assembly contigs with BWA-MEM. Preprint at https://arxiv.org/abs/1303.3997 (2013).

57. Li, H. et al. The sequence alignment/map format and SAMtools. Bioinformatics 25, 2078-2079 (2009).

58. Garcia-Alcalde, F. et al. Qualimap: evaluating next-generation sequencing alignment data. Bioinformatics 28, 2678-2679 (2012).

59. Arndt, D. et al. PHASTER: a better, faster version of the PHAST phage search tool. Nucleic Acids Res. 44, W16-W21 (2016).

60. Quinlan, A. R. BEDTools: the Swiss-Army tool for genome feature analysis. Curr. Protoc. Bioinformatics 47, 11.12.1-34 (2014).

61. Croucher, N. J. et al. Rapid phylogenetic analysis of large samples of recombinant bacterial whole genome sequences using Gubbins. Nucleic Acids Res. 43, e15 (2015).

62. Nguyen, L. T., Schmidt, H. A., von Haeseler, A. \& Minh, B. Q. IQ-TREE: a fast and effective stochastic algorithm for estimating maximum-likelihood phylogenies. Mol. Biol. Evol. 32, 268-274 (2015).

63. Letunic, I. \& Bork, P. Interactive Tree Of Life (iTOL) v4: recent updates and new developments. Nucleic Acids Res. 47, W256-W259 (2019).

64. Rambaut, A., Lam, T. T., Max Carvalho, L. \& Pybus, O. G. Exploring the temporal structure of heterochronous sequences using TempEst (formerly Path-O-Gen). Virus Evol. 2, vew007 (2016).

65. Bouckaert, R. et al. BEAST 2: a software platform for Bayesian evolutionary analysis. PLoS Comput. Biol. 10, e1003537 (2014).

66. Bouckaert, R. R. \& Drummond, A. J. bModelTest: Bayesian phylogenetic site model averaging and model comparison. BMC Evol. Biol. 17, 42 (2017).

67. Rambaut, A., Drummond, A. J., Xie, D., Baele, G. \& Suchard, M. A. Posterior summarization in Bayesian phylogenetics using Tracer 1.7. Syst. Biol. 67, 901-904 (2018).

68. Bouckaert, R. et al. BEAST 2.5: an advanced software platform for Bayesian evolutionary analysis. PLoS Comput. Biol. 15, e1006650 (2019).

69. Wick, R. R., Judd, L. M., Gorrie, C. L. \& Holt, K. E. Unicycler: resolving bacterial genome assemblies from short and long sequencing reads. PLoS Comput. Biol. 13, e1005595 (2017)

70. Gurevich, A., Saveliev, V., Vyahhi, N. \& Tesler, G. QUAST: quality assessment tool for genome assemblies. Bioinformatics 29, 1072-1075 (2013).

71. Seemann, T. Prokka: rapid prokaryotic genome annotation. Bioinformatics $\mathbf{3 0}$, 2068-2069 (2014).

72. Page, A. J. et al. Roary: rapid large-scale prokaryote pan genome analysis. Bioinformatics 31, 3691-3693 (2015).

73. Wu, Y., Lau, H. K., Lee, T., Lau, D. K. \& Payne, J. In silico serotyping based on whole-genome sequencing improves the accuracy of Shigella identification. Appl. Environ. Microbiol. 85 e00165-19 (2019).

74. Inouye, M. et al. SRST2: rapid genomic surveillance for public health and hospital microbiology labs. Genome Med. 6, 90 (2014).

75. Altschul, S. F. et al. Gapped BLAST and PSI-BLAST: a new generation of protein database search programs. Nucleic Acids Res. 25, 3389-3402 (1997).

76. Larsson, A. AliView: a fast and lightweight alignment viewer and editor for large datasets. Bioinformatics 30, 3276-3278 (2014).

77. Hildebrand, A., Remmert, M., Biegert, A. \& Soding, J. Fast and accurate automatic structure prediction with HHpred. Proteins 77 , 128-132 (2009).

78. Zimmermann, L. et al. A completely reimplemented MPI bioinformatics toolkit with a new HHpred server at its core. J. Mol. Biol. 430 , 2237-2243 (2018)

79. Song, Y. et al. High-resolution comparative modeling with RosettaCM. Structure 21, 1735-1742 (2013).

80. Yang, J. et al. Improved protein structure prediction using predicted interresidue orientations. Proc. Natl Acad. Sci. USA 117, 1496-1503 (2020).

81. Studer, G. et al. QMEANDisCo-distance constraints applied on model quality estimation. Bioinformatics 36, 2647 (2020).
82. Studer, G., Biasini, M. \& Schwede, T. Assessing the local structural quality of transmembrane protein models using statistical potentials (QMEANBrane). Bioinformatics 30, i505-i511 (2014).

83. Chen, Y. et al. PremPS: predicting the impact of missense mutations on protein stability. PLoS Comput. Biol. 16, e1008543 (2020).

84. Feldgarden, M. et al. Validating the AMRFinder tool and resistance gene database by using antimicrobial resistance genotype-phenotype correlations in a collection of isolates. Antimicrob. Agents Chemother. 63, e00483-19 (2019).

85. Conway, J. R., Lex, A. \& Gehlenborg, N. UpSetR: an R package for the visualization of intersecting sets and their properties. Bioinformatics 33, 2938-2940 (2017)

86. Hudzicki, J. Kirby-Bauer Disk Diffusion Susceptibility Test Protocol (American Society for Microbiol, 2009).

\section{Acknowledgements}

We thank the members of Baker group and $\mathrm{Lab} \mathrm{H}$ at the University of Liverpool, and R. Bacigalupe at KU Leuven for invaluable discussions; J. Hinton and B. P. Sepulveda for orchestrating the thermolysate shipping; S. Haldenby, M. Gemmell, R. Gregory and the Centre for Genomics Research, University of Liverpool for technical support; Dr I. Kasumba, J. Jones, S. Sen and J.-P.-Booth for preparing GEMS Shigella isolates for sequencing and antimicrobial testing. This work was supported by a UKRI MRC NIRG award (MR/R020787/1, KSB), the National Institute of Allergy and Infectious Diseases, National Institutes of Health, Department of Health and Human Services (U19AI110820, DR), and by both a Global Challenges Research Fund (GCRF) data and resources grant (BBS/OS/GC/000009D, NH) and the BBSRC Core Capability Grant to the Earlham Institute (BB/CCG1720/1, NH). Next-generation sequencing and library construction were delivered via the BBSRC National Capability in Genomics and Single Cell (BB/ CCG1720/1, NH) at Earlham Institute, by members of the Genomics Pipelines Group. K.S.B. was supported by a Wellcome Trust Clinical Research Career Development Award (106690/A/14/Z) and an Academy of Medical Sciences Springboard award (SBF002/1114), and is affiliated with the National Institute for Health Research Health Protection Research Unit (NIHR HPRU) in Gastrointestinal Infections at the University of Liverpool in partnership with Public Health England (PHE) and in collaboration with the University of Warwick. The views expressed herein are those of the author(s) and do not necessarily represent those of the NHS, the NIHR, the Department of Health and Social Care or Public Health England.

\section{Author contributions}

R.J.B. performed majority of the data analysis and interpretation of the results under the scientific guidance of K.S.B.; A.J.S. and D.J.R. performed in silico protein antigens modelling and prediction of the impacts of amino acid substitutions on protein stability; C.V.P. supported Bayesian Evolutionary Analysis by Sampling Trees; S.M.T. prepared and provided GEMS Shigella isolates and metadata and provided feedback on intermediary results; D.A.R. contributed to sample preparation; N.H. and R.L. generated sequencing data and conducted quality control for sequencing performed at the Earlham Institute; R.J.B. and K.S.B. drafted the manuscript. All authors contributed to editing of the manuscript.

\section{Competing interests}

The authors declare no competing interests.

\section{Additional information}

Extended data is available for this paper at https://doi.org/10.1038/s41564-021-01054-z.

Supplementary information The online version contains supplementary material available at https://doi.org/10.1038/s41564-021-01054-z.

Correspondence and requests for materials should be addressed to Kate S. Baker.

Peer review information Nature Microbiology thanks Rino Rappuoli and the other, anonymous, reviewer(s) for their contribution to the peer review of this work.

Reprints and permissions information is available at www.nature.com/reprints.

Publisher's note Springer Nature remains neutral with regard to jurisdictional claims in published maps and institutional affiliations.

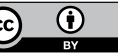

Open Access This article is licensed under a Creative Commons Attribution 4.0 International License, which permits use, sharing, adaptation, distribution and reproduction in any medium or format, as long as you give appropriate credit to the original author(s) and the source, provide a link to the Creative Commons license, and indicate if changes were made. The images or other third party material in this article are included in the article's Creative Commons license, unless indicated otherwise in a credit line to the material. If material is not included in the article's Creative Commons license and your intended use is not permitted by statutory regulation or exceeds the permitted use, you will need to obtain permission directly from the copyright holder. To view a copy of this license, visit http://creativecommons.org/licenses/by/4.0/.

(C) The Author(s) 2022 
A

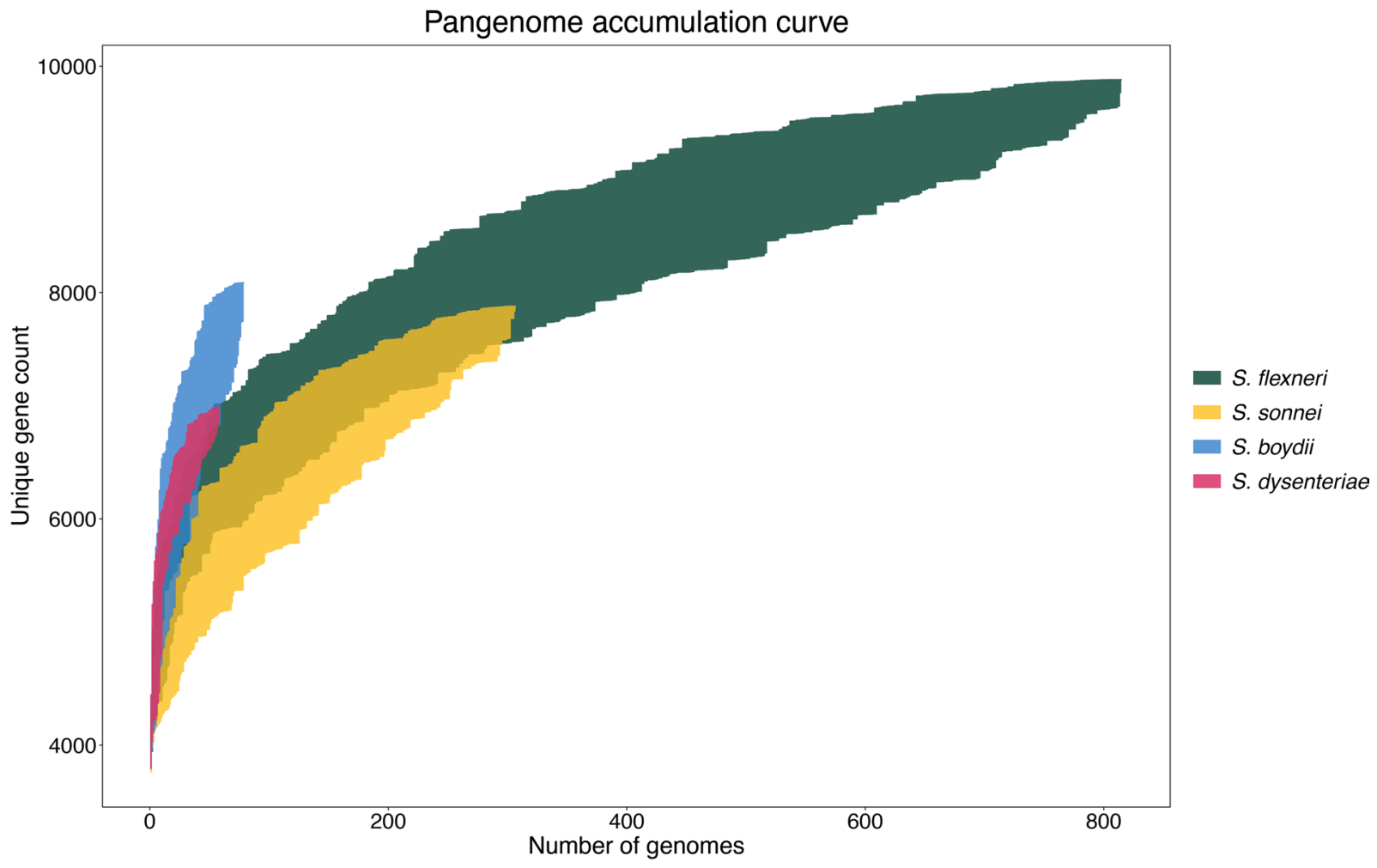

B

Pangenome accumulation curve

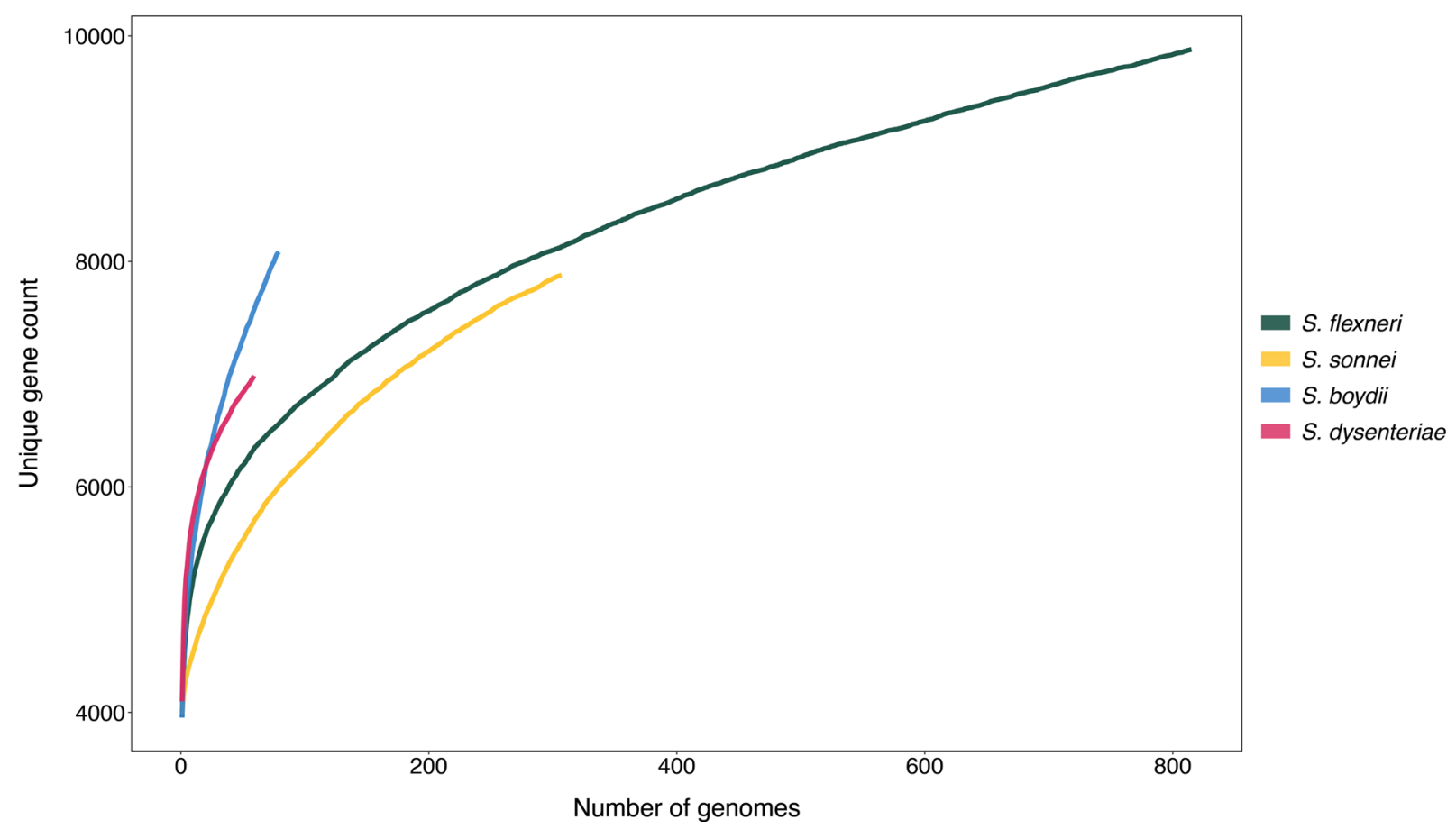

Extended Data Fig. 1 | See next page for caption. 
Extended Data Fig. 1 | Pangenome accumulation curve of S. flexneri, S. sonnei, S. boydii and S. dysenteriae. Each curve demonstrates the number of unique protein coding genes in the pangenome as a new genome is randomly added. Random permutation of the data were subsampled 100 times, in which genomes are subsampled without replacement at each iteration. The $x$-axis display the number of genomes and the $y$-axis shows the minimum and maximum range of unique gene count after each iteration in $(A)$ and the mean value in (B). 

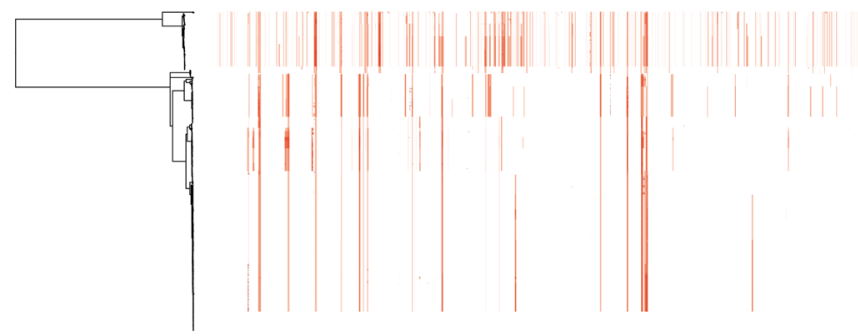

S. dysenteriae

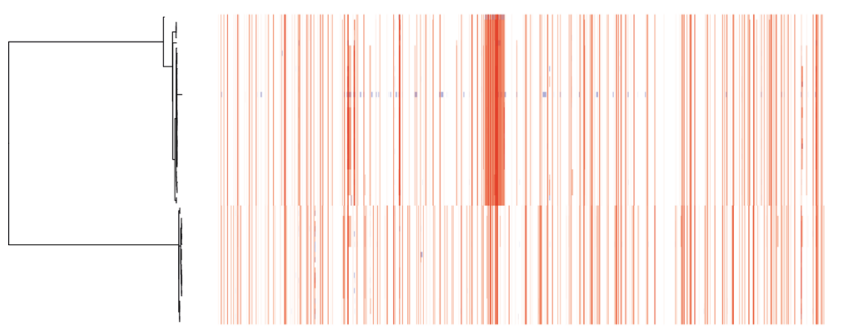

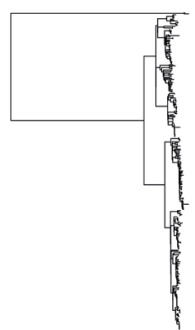

S. boydii
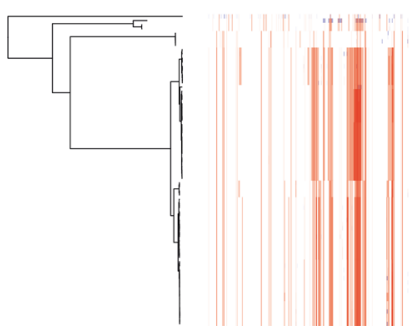

S. boydii

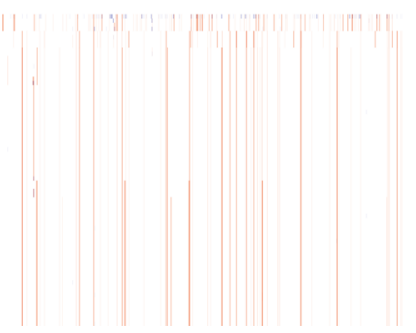

Extended Data Fig. 2 | Detection of recombination among Shigella genomes. ML phylogenetic tree of each species are displayed on the left. Coloured columns represents regions of putative recombination with elevated SNP density detected across the genome. Columns highlighted in red represents recombination detected in multiple isolates and blue represents recombination detected in a single isolate. 


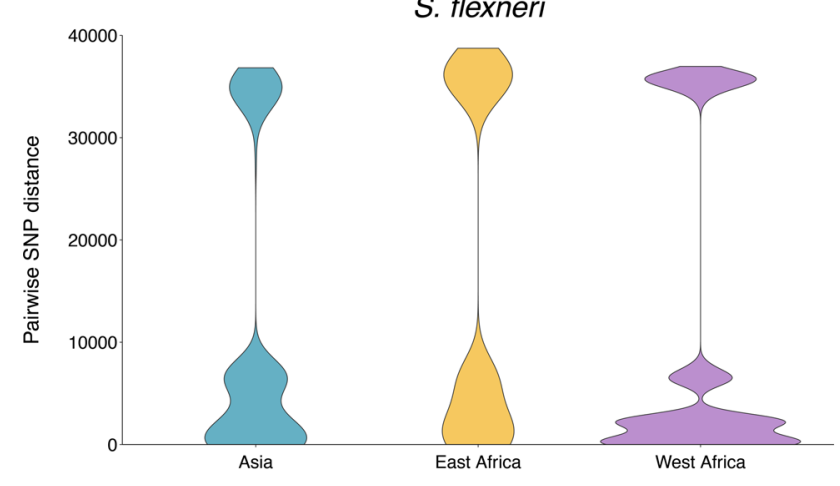

S. boydii

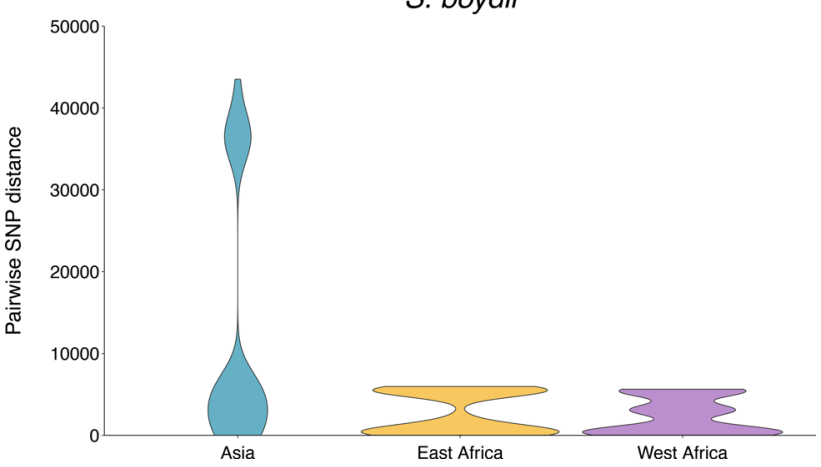

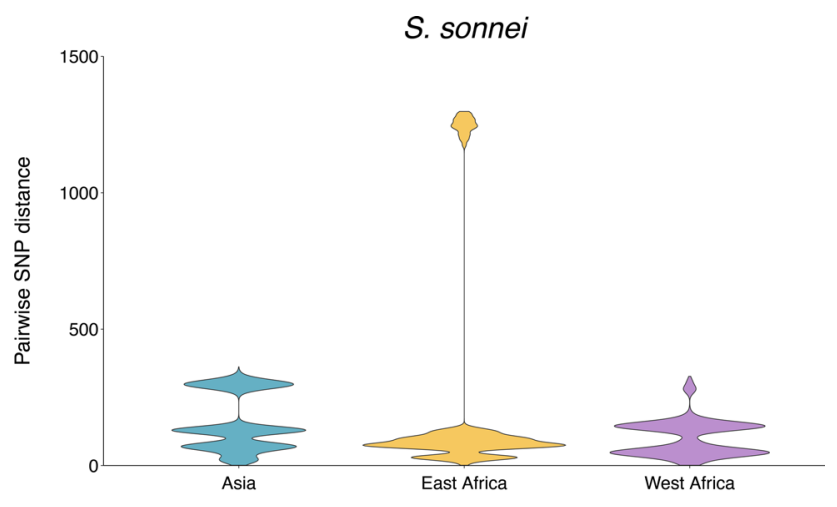

S. dysenteriae

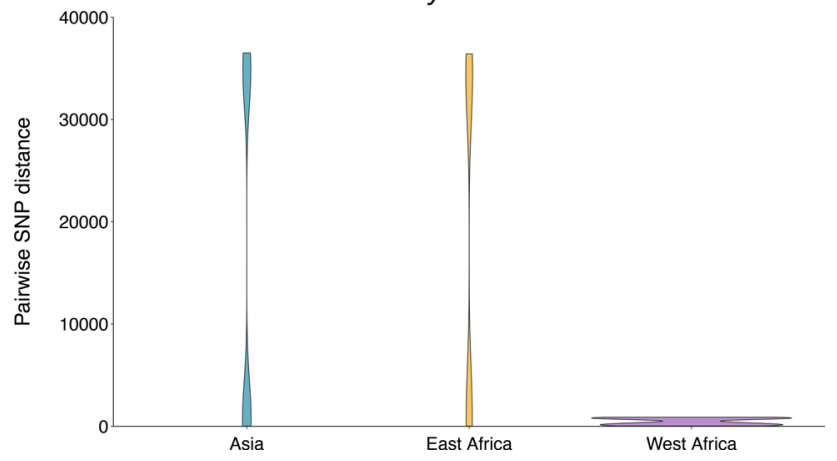

Extended Data Fig. 3 | Regional genomic diversity of GEMS Shigella. Comparison of regional genomic diversity of Shigella spp, as measured by pairwise core SNP distance ( $x$-axis) across GEMS study sites (Asia: Bangladesh, India and Pakistan; East Africa: Kenya and Mozambique; West Africa: The Gambia and Mali) for S. flexneri, S. sonnei, S. boydii and S. dysenteriae. 
S. flexneri
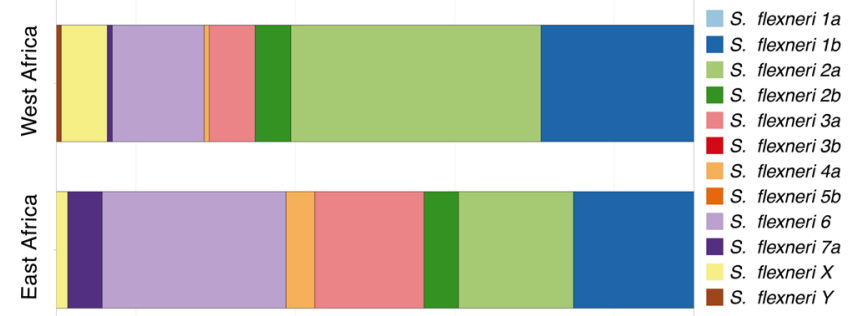

$\frac{-\pi}{2}$

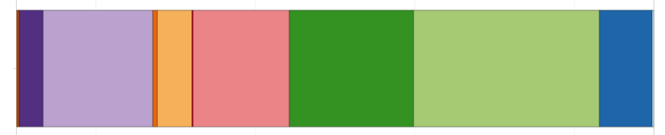

\section{S. dysenteriae}
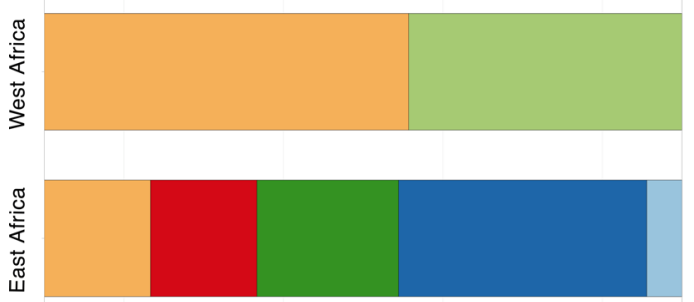

$\frac{-\pi}{4}$

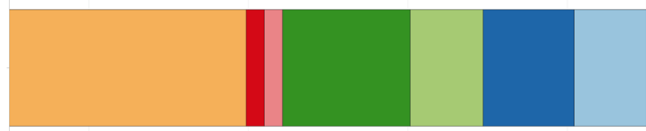

S. boydii

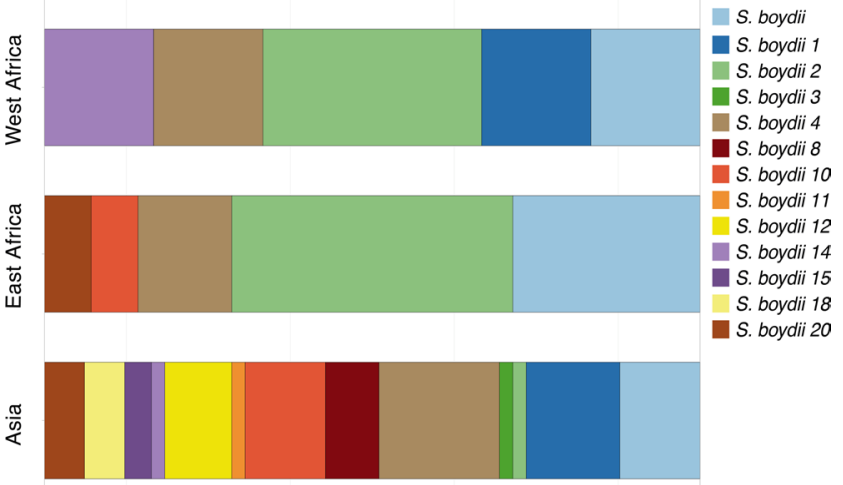

S. dysenteriae

S. dysenteriae 2

S. dysenteriae 3

S. dysenteriae 4

S. dysenteriae 9

S. dysenteriae 12

S. dysenteriae provisional 96-3162

Extended Data Fig. 4 | Regional serotypes/subserotypes diversity of GEMS Shigella. Regional serotypes/subserotypes diversity of Shigella spp across GEMS study sites for S. flexneri, S. boydii and S. dysenteriae. Each barplot represents the relative frequencies of serotypes/subserotypes at each region according to the inlaid key. 
A

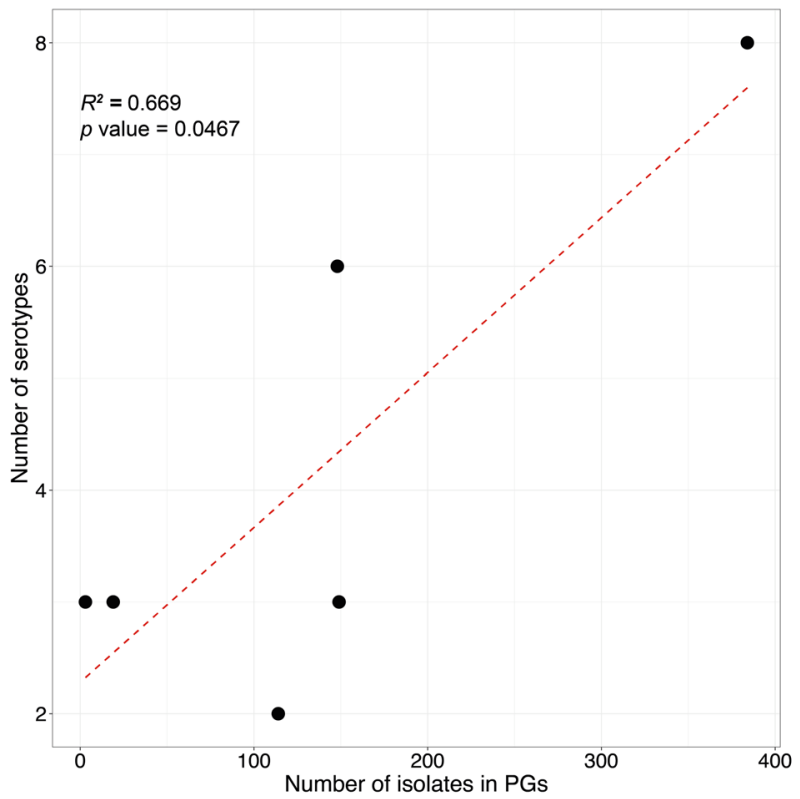

B

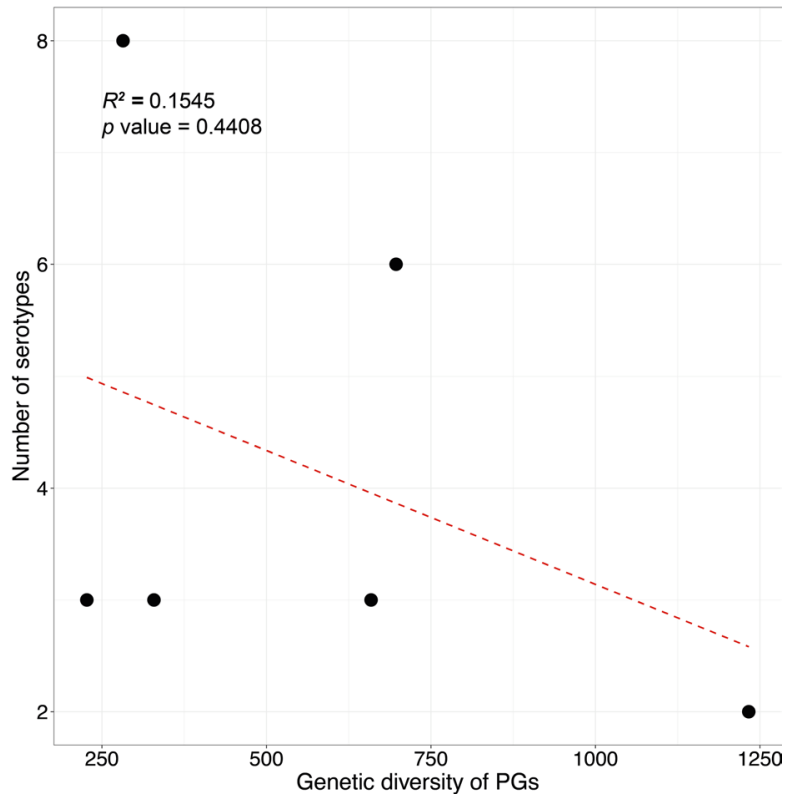

Extended Data Fig. 5 | Association of S. flexneri serotype diversity with different properties of a genomic subtype. Each of the six subtypes identified among S. flexneri (PG1-PG7 and Sf6), the number of different serotypes is displayed along the $y$-axis and plotted against (A) the number of isolates within the subtype and $(B)$ the genetic diversity of the subtype, as measured by pairwise core SNP distance and plotted along the $x$-axis. Linear regression analysis was performed to assess the association between serotype diversity and the different properties of subtypes. The regression coefficient of determination $\left(\mathrm{R}^{2}\right)$ and $p$-value are displayed on the top left of each plot. 
PG1

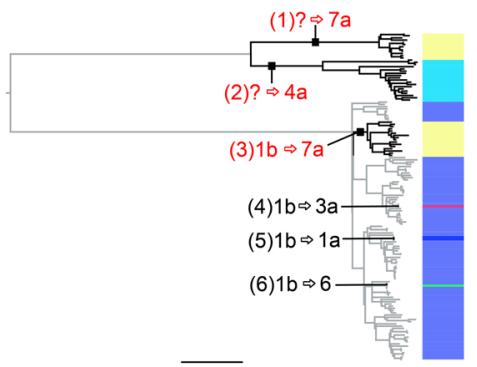

$\overline{200 \text { SNPs }}$

PG2

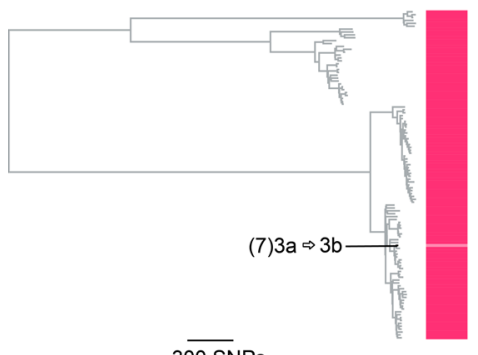

300 SNPS

PG7

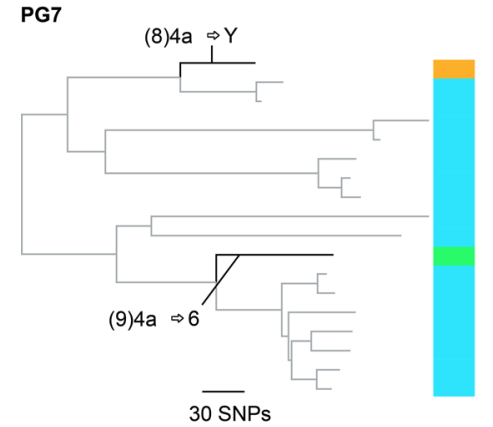

PG3

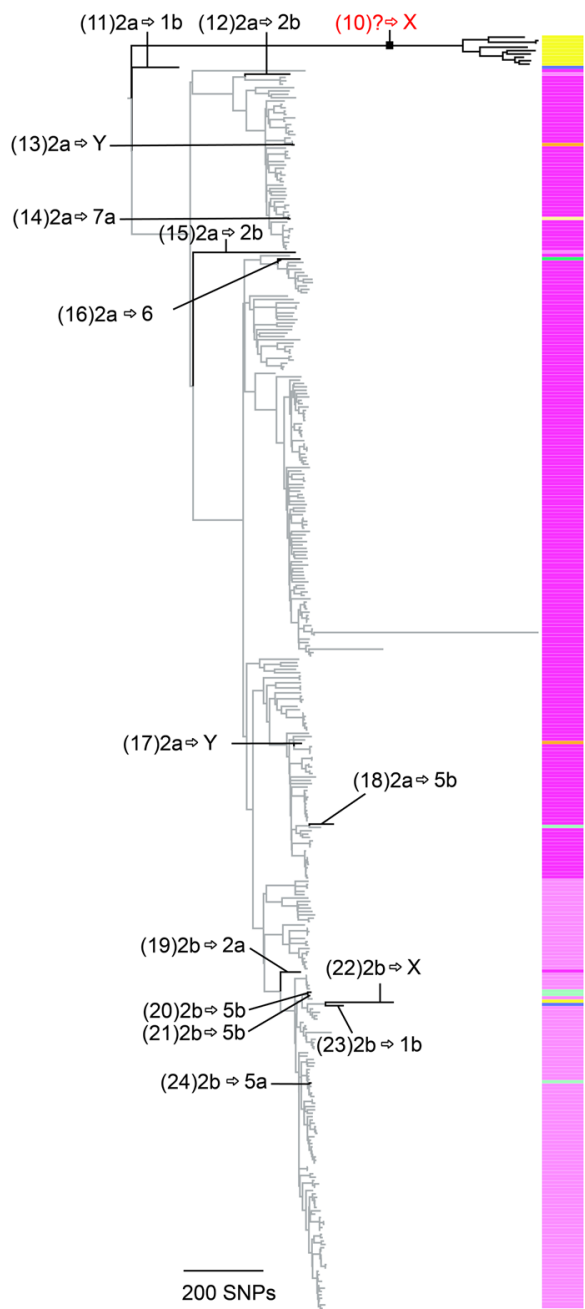

Sf6

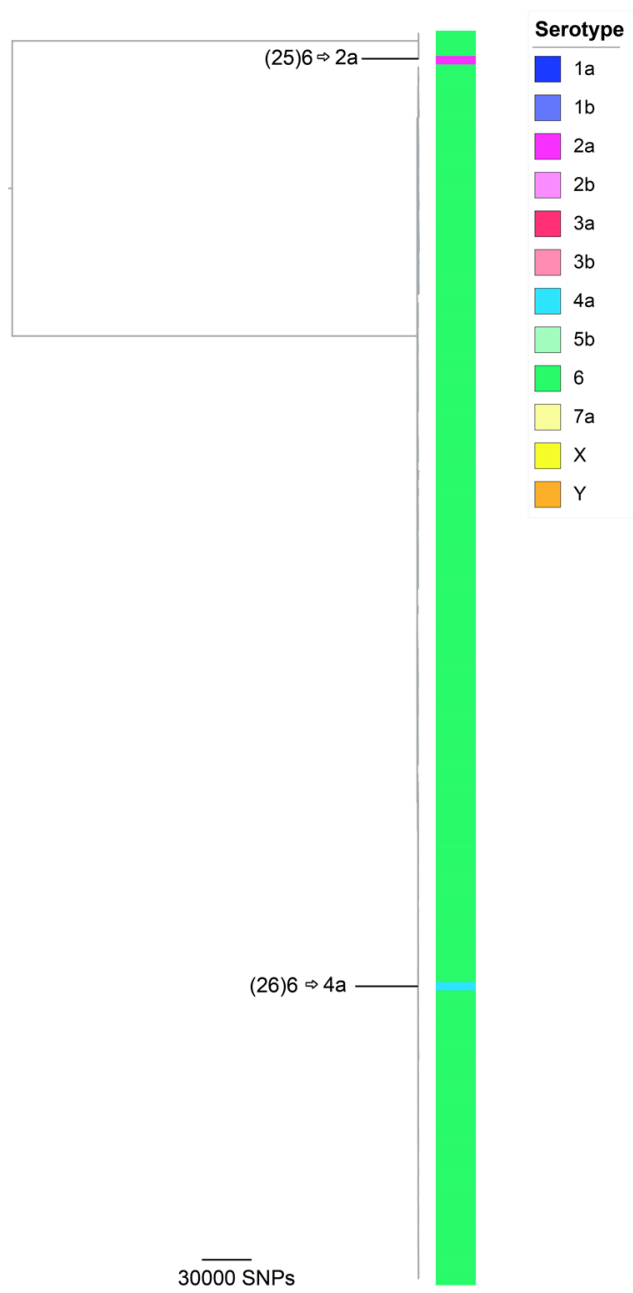

Extended Data Fig. 6 | Serotype switching events across S. flexneri genomic subtypes. ML phylogenetic tree of each subtype was generated based on core genome SNPS. Serotypes determined through biochemical serotyping are displayed on the right-hand side of each tree, and coloured according to the inlaid key. The 26 inferred serotype switching events occurring along the phylogenetic branches are labelled accordingly. Numbers inside each backets represents switch IDs, with further details provided in Supplementary Table 4. Where the dominant serotype cannot be determined, a question mark is displayed, indicating switch from unknown ancestral type. Serotype switching events resulting in more than two descendant isolates are highlighted in red. 


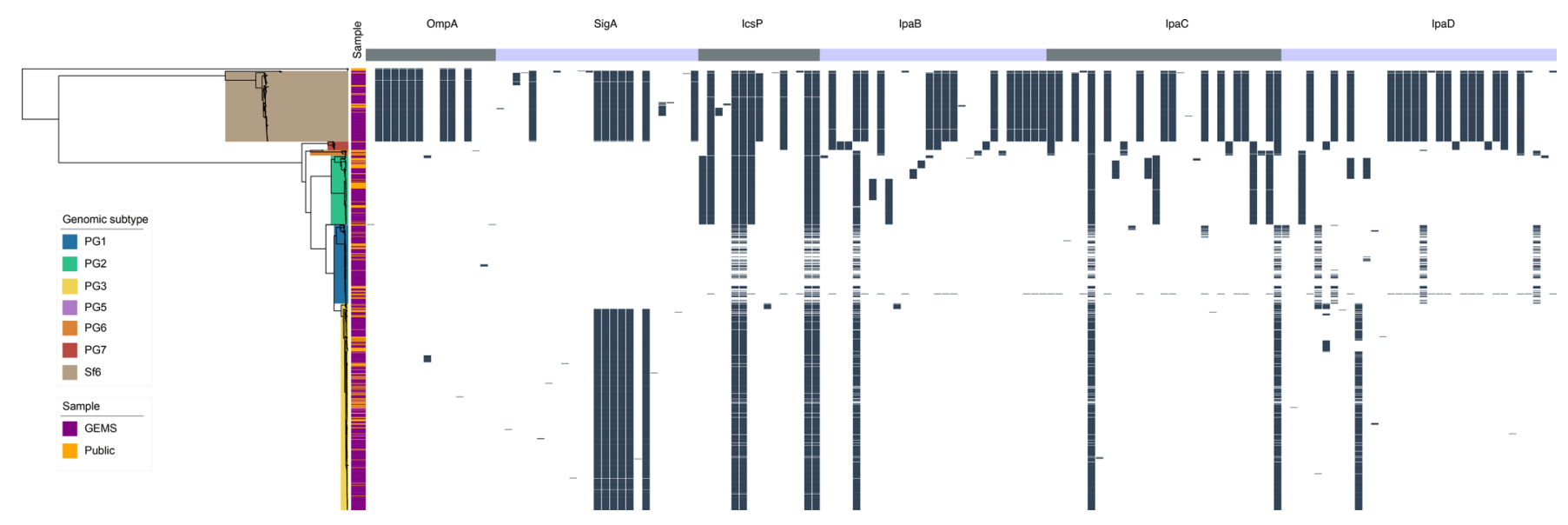

Extended Data Fig. 7 | Vaccine antigen variation among S. flexneri subtypes. ML phylogenetic tree of 806S. flexneri GEMS and 236 publicly available genomes, based on core genome SNPs is displayed on the left. The six subtypes identified among the population are highlighted in different colours according to the inlaid key. The alternating grey and purple colour blocks displayed above the top panel represents the six antigen vaccine candidates assessed in the current study. The matrix in the center demonstrates presence (in black) of aa variation for each antigen vaccine. Only variable sites are displayed. The full list of variable sites represented are available in Supplementary Table 8. 

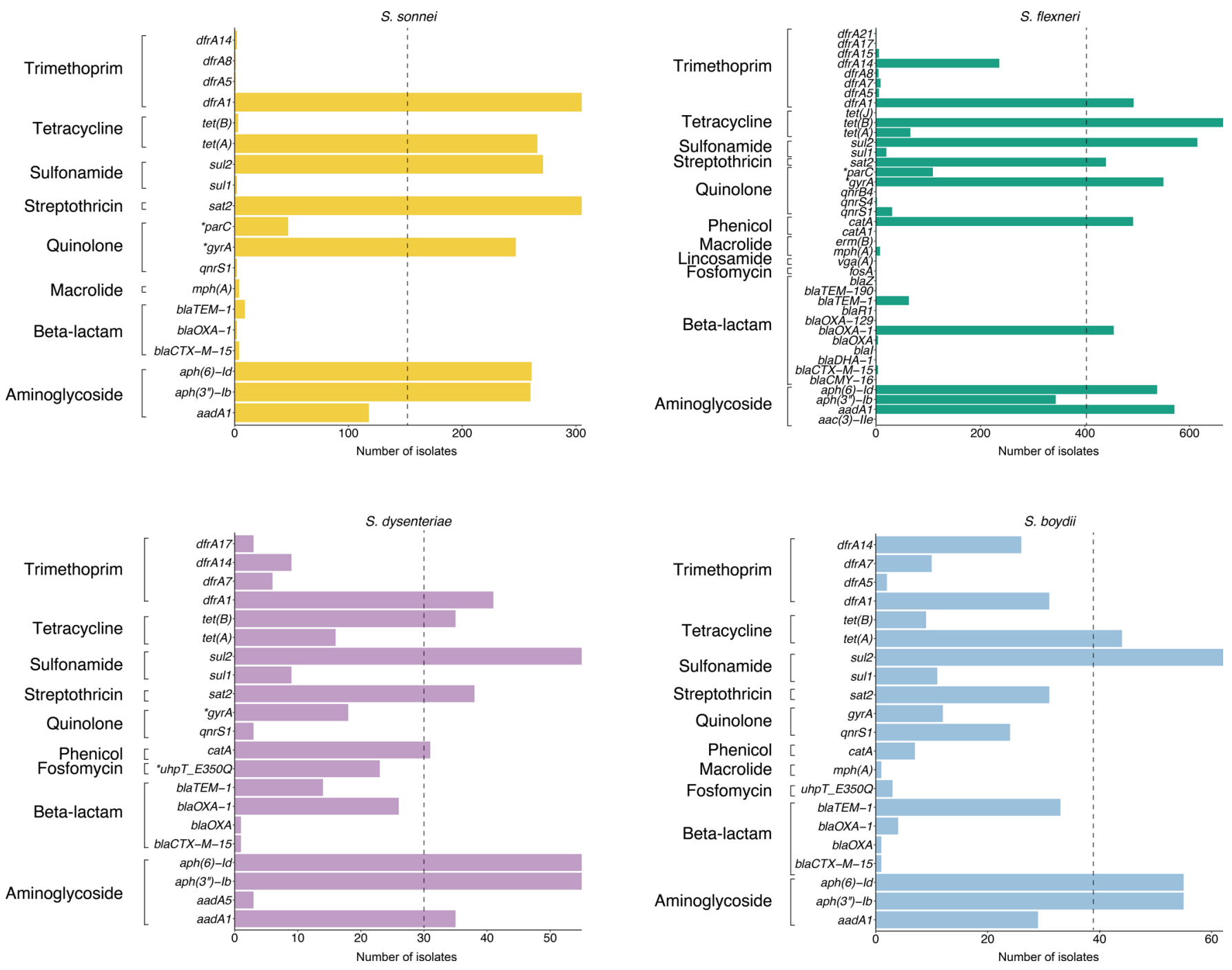

Extended Data Fig. 8 | Prevalence of genetic determinants conferring AMR among Shigella spp. Barplots shows the number of genetic determinants detected in S. sonnei, S. flexneri, S. dysenteriae and S. boydii isolates that confer resistance or reduced susceptibility to various antimicrobials. Genes and point mutations (indicated with an asterisk) are plotted along the $y$-axis and grouped by drug class (displayed on the left). The dashed lines highlight genetic determinants identified in half or more of the isolates for each species. 
A

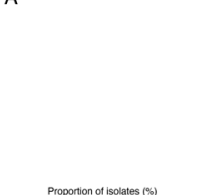

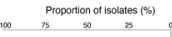

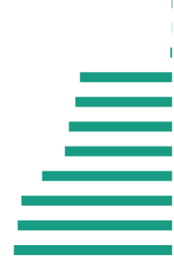<smiles>CC1CC1C</smiles>

s. flexneri

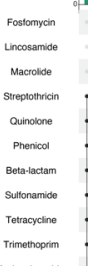

C

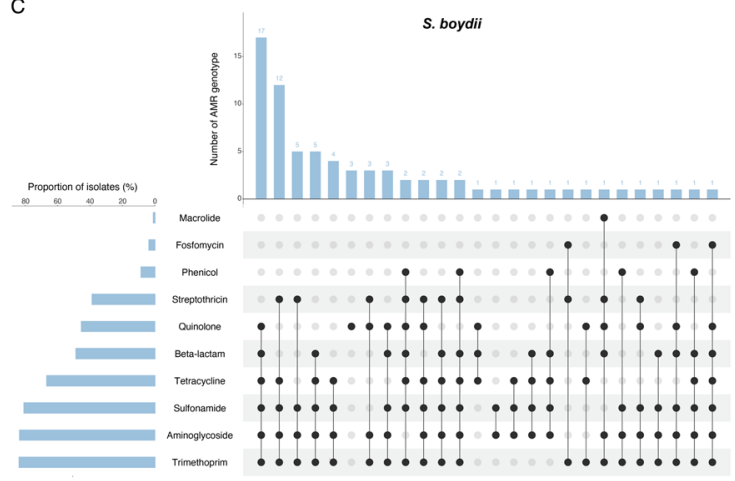

B

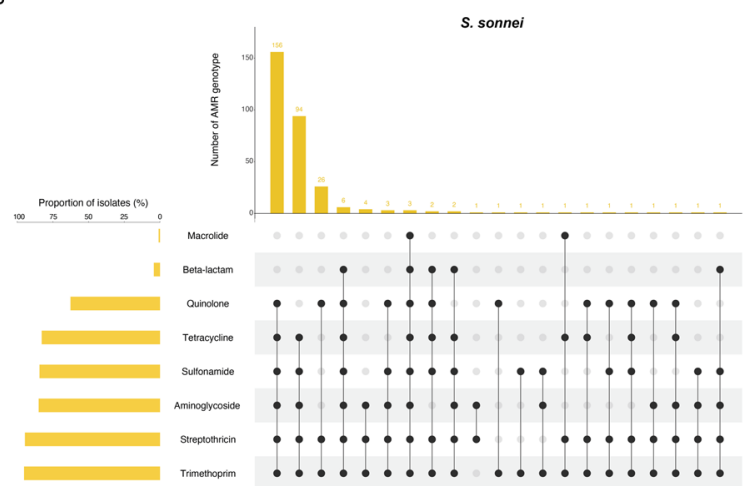

D

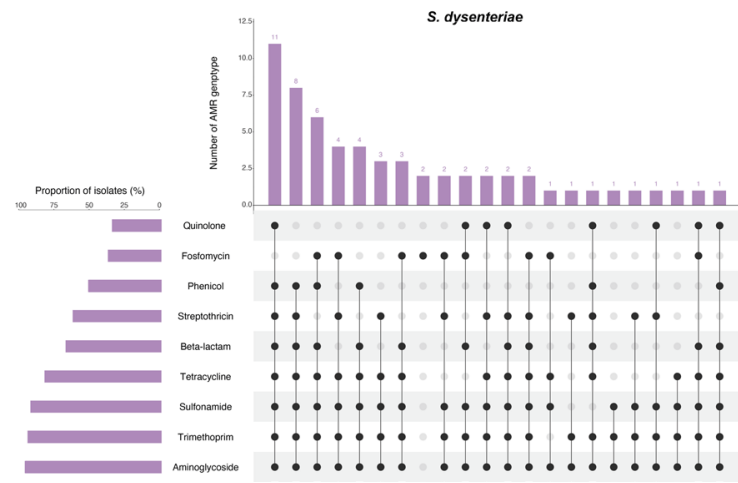

Extended Data Fig. 9 | Diversity of AMR genotype resistance profiles. UpSet plots illustrate the AMR genotype resistance profiles for (A) S. flexneri, (B) S. sonnei, (C) S. boydii and (D) S. dysenteriae. Genotypic AMR profiles are shown in the combination matrix in the center panel. Each column represents a unique genotypic profile, where each black dot represents presence of a genetic determinant conferring resistance or reduced susceptibility to a drug class (displayed on the left). The vertical the barplot above the matrix displays the number of isolates with a particular profile, with the exact number of isolates displayed above each bar. The horizontal barplot on the left of the matrix illustrates the proportion of isolates containing AMR genetic determinants associated with a drug class. 
A

\section{S. flexneri $2 \mathrm{a}(n=45)$}

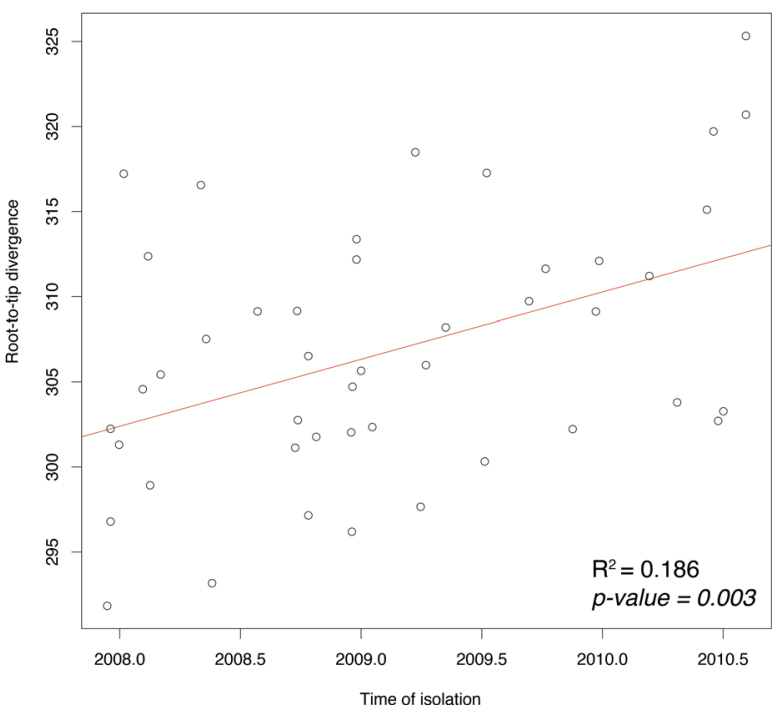

B

S. flexneri $2 b(n=99)$

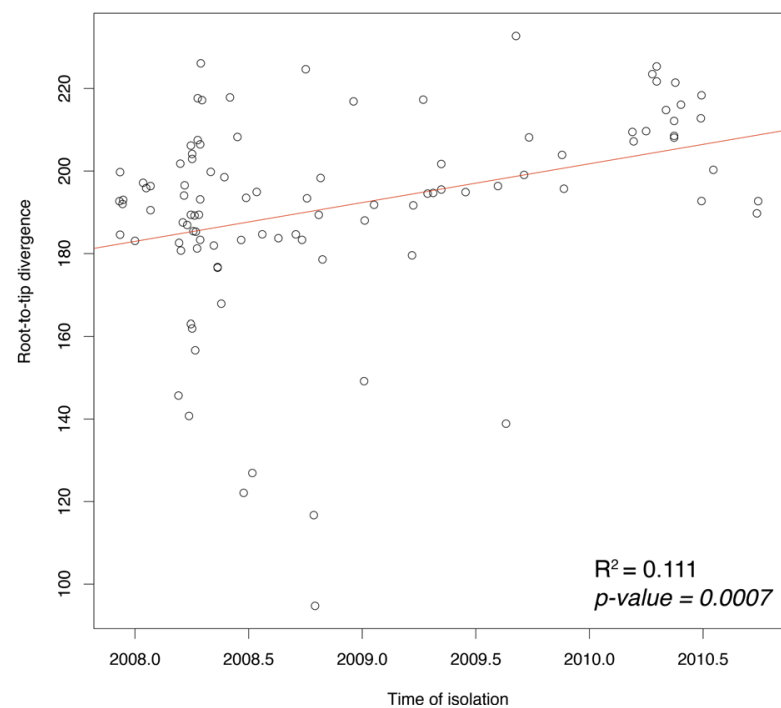

Extended Data Fig. 10 | Temporal phylogenetic signal for S. flexneri. Correlation between isolate sampling time in months ( $x$-axis) and phylogenetic root-to-tip divergence ( $y$-axis), as estimated by TempEst based on ML phylogeny of each subclade. The two datasets correspond to $S$. flexneri serotype $2 a$ isolates belonging to node $A(A)$ and $S$. flexneri serotype $2 b$ isolates belonging to node $B(B)$ from PG3 in Supplementary Fig. 12. The linear regression line is coloured in red, with the coefficient of determination $\left(R^{2}\right)$ and $p$-value displayed for each plot. 


\section{natureportfolio}

Corresponding author(s): Kate S. Baker

Last updated by author(s): 2021-12-03

\section{Reporting Summary}

Nature Portfolio wishes to improve the reproducibility of the work that we publish. This form provides structure for consistency and transparency in reporting. For further information on Nature Portfolio policies, see our Editorial Policies and the Editorial Policy Checklist.

\section{Statistics}

For all statistical analyses, confirm that the following items are present in the figure legend, table legend, main text, or Methods section.

n/a Confirmed

$\square$ The exact sample size $(n)$ for each experimental group/condition, given as a discrete number and unit of measurement

Х $\square$ A statement on whether measurements were taken from distinct samples or whether the same sample was measured repeatedly

$\square$ The statistical test(s) used AND whether they are one- or two-sided

Only common tests should be described solely by name; describe more complex techniques in the Methods section.

$\searrow$ A description of all covariates tested

\ $\square$ A description of any assumptions or corrections, such as tests of normality and adjustment for multiple comparisons

$\triangle$ A full description of the statistical parameters including central tendency (e.g. means) or other basic estimates (e.g. regression coefficient)

$\bigotimes$ AND variation (e.g. standard deviation) or associated estimates of uncertainty (e.g. confidence intervals)

$\varnothing$ For null hypothesis testing, the test statistic (e.g. $F, t, r$ ) with confidence intervals, effect sizes, degrees of freedom and $P$ value noted Give $P$ values as exact values whenever suitable.

$\square$ \For Bayesian analysis, information on the choice of priors and Markov chain Monte Carlo settings

$\bigotimes \square$ For hierarchical and complex designs, identification of the appropriate level for tests and full reporting of outcomes

$\bigotimes \square$ Estimates of effect sizes (e.g. Cohen's $d$, Pearson's $r$ ), indicating how they were calculated

Our web collection on statistics for biologists contains articles on many of the points above.

\section{Software and code}

Policy information about availability of computer code

Data collection Microsoft Excel for Mac v16.50

Data analysis Trimmomatic v0.38, FastQC v0.11.6, MultiQC v1.7, BWA mem v0.7.17, SAMtools v1.9-47, Picard v2.21.1-SNPSHOT, QualiMap v2.2.2,

Bcftools v1.9-80, PHASTER (https://phaster.ca/), BEDTools v2.28.0, Gubbins v2.3.4, IQ-TREE v2.0-rc2, iTOL v6.1.1, snp-dists v0.7.0, TempEst v1.5.3, BEAUTI v2.6.1, BEAST2 v2.6.1, bModelTest, Tracer v1.7.1, LogCombiner v2.6.1, TreeAnnotator v2.6.0, Unicycler v0.4.7, QUAST v5.0.2,

Prokka v1.13.3, Roary v3.12.0, Vegan v2.5-7, ShigaTyper v1.0.6, SRST2 v2, screen assembly (https://github.com/shimbalama/screen_assembly), AliView v1.26, HHPred, RosettaCM source relase-188, trRosetta, QMEAN v4.2.0, QMEANbrane v4.2.0, QMEANDisCo v4.2.0, PremPS, AMRFinderPlus v3.9.3, UpSetR v2.1.3, R v4.0.3 MedCalc's odds ratio calculator v20

For manuscripts utilizing custom algorithms or software that are central to the research but not yet described in published literature, software must be made available to editors and reviewers. We strongly encourage code deposition in a community repository (e.g. GitHub). See the Nature Portfolio guidelines for submitting code \& software for further information.

\section{Data}

Policy information about availability of data

All manuscripts must include a data availability statement. This statement should provide the following information, where applicable:

- Accession codes, unique identifiers, or web links for publicly available datasets

- A description of any restrictions on data availability

- For clinical datasets or third party data, please ensure that the statement adheres to our policy 
from GenBank (https://www.ncbi.nlm.nih.gov/genbak/), Sequence Read Archive (https://www.ncbi.nlm.nih.gov/sra), European Nucleotide Archive (https://www.ebi.ac.uk/ ena) or EnteroBase (https://enterobase.warwick.ac.uk), with accession numbers listed in Supplementary Table 3. Phylogenetic trees and antigen protein models have been deposited in FigShare: (DOI:10.6084/m9.figshare.14743833).

\section{Field-specific reporting}

Please select the one below that is the best fit for your research. If you are not sure, read the appropriate sections before making your selection. $\bigotimes$ Life sciences $\quad \square$ Behavioural \& social sciences $\quad \square$ Ecological, evolutionary \& environmental sciences

\section{Life sciences study design}

All studies must disclose on these points even when the disclosure is negative.

Sample size Samples from this study were derived from stool samples of children from the Global Enteric Multicenter Study (GEMS).

All isolates which were confirmed to be Shigella through biochemical tests and agglutination with antisera were whole genome sequenced, resulting in a sample size of 1,344

Data exclusions Any sample which failed quality control of sequence reads were excluded. Specifically, any samples that was identified as a serotype other than Shigella following computational typing of genome sequence data and phylogenetic analysis were excluded. Samples with a mean sample depth of coverage $<10 x$ and samples with total assembly size outside the range of $<4 \mathrm{Mbp}$ and $>6.4 \mathrm{Mbp}$ were excluded.

Replication

Robust maximum likelihood phylogenetic trees were generated with 1000 bootstrap replicates to determine branch support. Bayesian evolutionary analysis was run. on five independent chains, each of length $250,000,000$. Replicates were well supported and individual branch support is available embedded in the tree files provided in FigShare repository 10.6084/m9.figshare. 14743833

Randomization

The GEMS Shigella isolates represent a systematic collection of bacteria taken during a previously described (and appropriately randomised) case-control study. Details of randomisation during the original study can be found in the methodological paper under PubMedCentral accession PMC3502307.

Blinding

\section{Reporting for specific materials, systems and methods}

We require information from authors about some types of materials, experimental systems and methods used in many studies. Here, indicate whether each material, system or method listed is relevant to your study. If you are not sure if a list item applies to your research, read the appropriate section before selecting a response.

\begin{tabular}{|c|c|}
\hline $\mathrm{n} / \mathrm{a}$ & Involved in the study \\
\hline$\bigotimes$ & Antibodies \\
\hline Х & Eukaryotic cell lines \\
\hline Х & Palaeontology and archaeology \\
\hline$\bigotimes$ & Animals and other organisms \\
\hline$\bigotimes$ & Human research participants \\
\hline Х & Clinical data \\
\hline$\bigotimes$ & Dual use research of concern \\
\hline
\end{tabular}

\begin{tabular}{l|l}
\multicolumn{2}{l}{ Methods } \\
\hline n/a & Involved in the study \\
\hline & $\square$ ChIP-seq \\
$\varnothing$ & $\square$ Flow cytometry \\
$\varnothing$ & $\square$ MRI-based neuroimaging
\end{tabular}

